LBNL-56403

\title{
An Overview of Alternative Fossil Fuel Price and Carbon Regulation Scenarios
}

\author{
Prepared for the \\ Office of Planning, Budget, and Analysis \\ Assistant Secretary for Energy Efficiency and Renewable Energy \\ U.S. Department of Energy
}

Principal Authors

Ryan Wiser and Mark Bolinger

Ernest Orlando Lawrence Berkeley National Laboratory

1 Cyclotron Road, MS 90R4000

Berkeley CA 94720-8136

October 2004

The work described in this paper was funded by the Assistant Secretary of Energy Efficiency and Renewable Energy, Office of Planning, Budget, and Analysis of the U.S. Department of Energy under Contract No. DE-AC03-76SF00098. 



\section{Acknowledgements}

The work described in this report was funded by the Assistant Secretary of Energy Efficiency and Renewable Energy, Office of Planning, Budget, and Analysis of the U.S. Department of Energy under Contract No. DE-AC03-76SF00098. The authors would especially like to thank Sam Baldwin, Mary Beth Zimmerman, and Brian Unruh of the U.S. Department of Energy for supporting this work. Appreciation also goes to Chris Marnay (LBNL), Chip Friley (BNL), Susan Holte (EIA), Scott Hassell (DOE), and Frances Wood (OnLocation, Inc.) for reviewing earlier versions of this report.

\section{Disclaimer}

This document was prepared as an account of work sponsored by the United States Government. While this document is believed to contain correct information, neither the United States Government nor any agency thereof, nor The Regents of the University of California, nor any of their employees, makes any warranty, express or implied, or assumes any legal responsibility for the accuracy, completeness, or usefulness of any information, apparatus, product, or process disclosed, or represents that its use would not infringe privately owned rights. Reference herein to any specific commercial product, process, or service by its trade name, trademark, manufacturer, or otherwise, does not necessarily constitute or imply its endorsement, recommendation, or favoring by the United States Government or any agency thereof, or The Regents of the University of California. The views and opinions of authors expressed herein do not necessarily state or reflect those of the United States Government or any agency thereof or The Regents of the University of California. 



\section{Table of Contents}

Acknowledgements ................................................................................................................

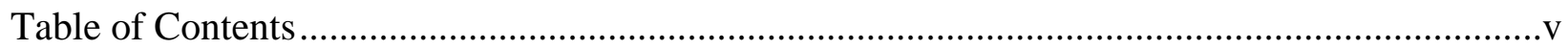

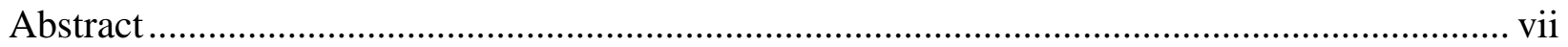

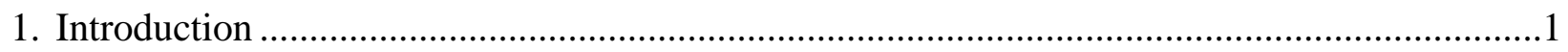



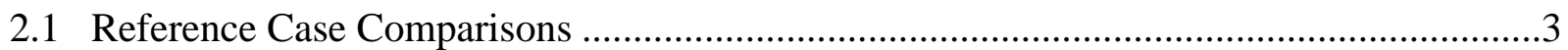

2.2 EIA High Gas Price Scenarios .........................................................................................

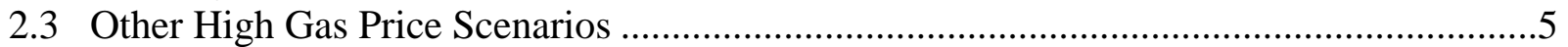

2.4 Scenarios from Utility Integrated Resource Plans .................................................................

2.5 Past EIA Forecast Accuracy ……………………......................................................11

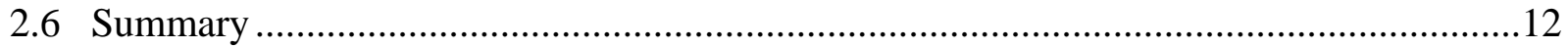

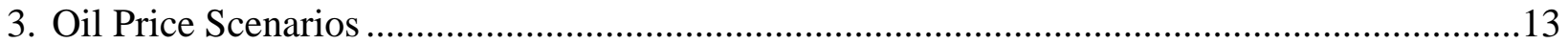

3.1 Reference Case Comparisons ……………………........................................................13

3.2 EIA High Oil Price Scenarios ..........................................................................................14

3.3 Other High Oil Price Scenarios....................................................................................15

3.4 Past EIA Forecast Accuracy …………………….....................................................16



4. Carbon Regulation Scenarios ...........................................................................................19

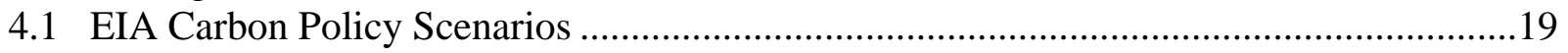

4.2 Other Domestic Carbon Reduction Scenarios ..............................................................22

4.3 International Comparisons .............................................................................................2

4.4 Carbon Scenarios in Utility Integrated Resource Planning ..............................................25

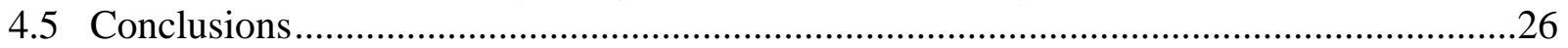

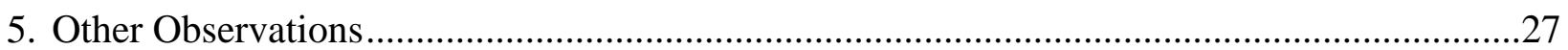





\begin{abstract}
The benefits of the Department of Energy's research and development (R\&D) efforts have historically been estimated under business-as-usual market and policy conditions. In recognition of the insurance value of R\&D, however, the Office of Energy Efficiency and Renewable Energy (EERE) and the Office of Fossil Energy (FE) have been exploring options for evaluating the benefits of their R\&D programs under an array of alternative futures.
\end{abstract}

More specifically, an FE-EERE Scenarios Working Group (the Working Group) has proposed to EERE and FE staff the application of an initial set of three scenarios for use in the Working Group’s upcoming analyses: (1) a Reference Case Scenario, (2) a High Fuel Price Scenario, which includes heightened natural gas and oil prices, and (3) a Carbon Cap-and-Trade Scenario. The immediate goal is to use these scenarios to conduct a pilot analysis of the benefits of EERE and FE R\&D efforts.

In this report, the two alternative scenarios being considered by EERE and FE staff - carbon capand-trade and high fuel prices - are compared to other scenarios used by energy analysts and utility planners. The report also briefly evaluates the past accuracy of fossil fuel price forecasts.

We find that the natural gas prices through 2025 proposed in the FE-EERE Scenarios Working Group's High Fuel Price Scenario appear to be reasonable based on current natural gas prices and other externally generated gas price forecasts and scenarios. If anything, an even more extreme gas price scenario might be considered. The price escalation from 2025 to 2050 within the proposed High Fuel Price Scenario is harder to evaluate, primarily because few existing forecasts or scenarios extend beyond 2025, but, at first blush, it also appears reasonable.

Similarly, we find that the oil prices originally proposed by the Working Group in the High Fuel Price Scenario appear to be reasonable, if not conservative, based on: (1) the current forward market for oil, (2) current oil prices, (3) externally generated oil price forecasts, and (4) the historical difficulty in accurately forecasting oil prices. Overall, a spread between the FE-EERE High Oil Price and Reference scenarios of well over $\$ 8 / \mathrm{bbl}$ is supported by the literature.

We conclude that a wide range of carbon regulation scenarios are possible, especially within the time frame considered by EERE and FE (through 2050). The Working Group’s Carbon Cap-\&Trade Scenario is found to be less aggressive than many Kyoto-style targets that have been analyzed, and similar in magnitude to the proposed Climate Stewardship Act. The proposed scenario is more aggressive than some other scenarios found in the literature, however, and ignores carbon banking and offsets and does not allow nuclear power to expand. We are therefore somewhat concerned that the stringency of the proposed carbon regulation scenario in the 2010 to 2025 period will lead to a particularly high estimated cost of carbon reduction. As described in more detail later, we encourage some flexibility in the Working Group's ultimate implementation of the Carbon Cap-and-Trade Scenario.

We conclude by identifying additional scenarios that might be considered in future analyses, describing a concern with the proposed specification of the High Fuel Price Scenario, and highlighting the possible difficulty of implementing extreme scenarios with current energy modeling tools. 



\section{Introduction}

The benefits of the Department of Energy's research and development (R\&D) efforts have historically been estimated only under business-as-usual market and policy conditions. In recognition of the insurance, or options, value of R\&D efforts, however, the Office of Energy Efficiency and Renewable Energy (EERE) and the Office of Fossil Energy (FE) have been jointly exploring options for evaluating the benefits of their R\&D efforts under an array of alternative futures. Specifically, alternative scenarios will be used to evaluate R\&D efforts under different market conditions, where the benefits of $R \& D$ within each scenario will be measured as the difference between a baseline (no R\&D program) case and a program (R\&D) case. ${ }^{1}$

As part of that effort, on July 30, 2004, the FE-EERE Scenarios Working Group (the Working Group) proposed to EERE and FE Staff the application of an initial set of three scenarios for use in the Working Group's upcoming analyses. The Working Group's immediate goal is to use those scenarios to conduct a pilot analysis of the benefits of EERE and FE R\&D efforts. Eventually it is hoped that routine analyses can be conducted under a broad range of possible future market conditions, and a more comprehensive evaluation of R\&D benefits derived.

The Working Group proposed three specific scenarios:

- Reference Case Scenario

- Carbon Cap-and-Trade Scenario

- High Fuel Price Scenario, which includes heightened natural gas and oil prices

The two alternative scenarios described by the Working Group, the Carbon Cap-and-Trade Scenario and the High Fuel Price Scenario, would ideally be benchmarked against (i.e., compared to) other scenarios commonly used among energy analysts. Such benchmarking would help to support whatever scenarios are ultimately chosen by FE and EERE management, and to assess how aggressive FE-EERE scenarios might be while still maintaining credibility.

This paper begins that benchmarking exercise. Specifically, we compile information on scenarios used in other analyses, focusing on natural gas price, oil price, and carbon regulation scenarios. While we have not sought to be exhaustive in our review, we do summarize a reasonably wide range of sources.

The following criteria have been used to loosely judge whether to include a particular study:

- Are the scenarios directly relevant and comparable to the proposed FE-EERE scenarios?

- Are the scenarios appropriately geographically focused?

- Are the scenarios sufficiently long-term?

- Are the scenarios from credible third-party "citable" sources?

- Are the scenarios sufficiently recent to be of maximum value?

\footnotetext{
${ }^{1}$ In other words, there will likely be a change in the technology mix in both the baseline case and the program case due to the alternative market conditions present in each scenario.
} 
We also briefly look at the past forecast accuracy of Energy Information Administration's (EIA) Annual Energy Outlook (AEO) projections for natural gas and oil prices, as we believe this also provides useful information on the plausible range of scenarios for these two variables. We note that a literature assessing energy model forecast accuracy has developed, much of which notes that energy models typically underestimate (or worse, ignore) uncertainties. ${ }^{2}$ This is also a key finding from an earlier study commissioned by EERE. ${ }^{3}$

We conclude this paper with some final observations on the scenarios approach to R\&D benefits estimation, and the FE-EERE Scenarios Working Group proposal.

\footnotetext{
${ }^{2}$ See, e.g., (a) Craig, P., Gadgil, A, and J. Koomey. 2002. "What Can History Teach Us? A Retrospective Examination of Long-Term Energy Forecasts for the United States." Annual Review of Energy and the Environment, 27: 83-188. (b) Sanstad, A. Laitner, J., and J. Koomey. 2004. "Back to the Future: Long-Range U.S. Energy Price and Quantity Projections in Retrospect. Draft Working Paper. (c) Koomey, J., P. Craig, A. Gadgil and D. Lorenzetti. 2003. “Improving Long-Range Energy Modeling: A Plea for Historical Retrospectives.” The Energy Journal, 24 (4): 75-92. (d) Shlyakhter, A., Kammen, D., Broido, C and R. Wilson. 1994. "Quantifying the Credibility of Energy Projections from Trends in Past Data: The US Energy Sector.” Energy Policy: 119-128. (e) Laitner, J., DeCanio, S., Koomey, J. and A. Sanstad. 2003. "Room for Improvement: Increasing the Value of Energy Modeling for Policy Analysis.” Utilities Policy, 11: 87-94.

${ }^{3}$ Silberglitt, R. and A. Hove. 2000. "Scenario Analysis." Presented at the E-Vision 2000 Conference. October 1113. Washington, D.C. For additional scenario review work conduced by EERE contractors, see Aabakken, J. and W. Short. 2003. "Domestic Energy Scenarios.” NREL/TP-620-32742. Golden, Colorado: National Renewable Energy Laboratory.
} 


\section{Natural Gas Price Scenarios}

Among actively traded commodities, natural gas ranks as one of the most volatile-priced. ${ }^{4}$ Future natural gas prices are also highly uncertain, and multiple price path scenarios are possible. In its memorandum dated July 30, 2004, the FE-EERE Scenarios Working Group proposed a High Fuel Price Scenario wherein natural gas wellhead prices (in 2002 \$ Mcf) reach $\$ 5.00$ by 2015, $\$ 5.50$ by 2025, and $\$ 7.50$ by 2050. In comparison, the Reference Case Scenario proposed in that memo (which is simply EIA's reference case as of April 9, 2004) shows gas prices reaching \$4.43 in 2025 (the ultimate year of the EIA forecast). Thus, in 2025, natural gas wellhead prices in the proposed High Fuel Price Scenario are \$1.07/Mcf above gas prices proposed by the Working Group in its Reference Case Scenario.

In this section we benchmark the natural gas prices contained in the proposed High Fuel Price Scenario against other such scenarios that have recently been externally developed. ${ }^{5}$ Where possible, we report not only the absolute price level of each scenario, but also the degree to which any high gas price scenario exceeds the corresponding "base-case" scenario, comparing that differential to the gap between the Working Group’s proposed High Fuel Price and Reference Case scenarios. This differential is relevant because the various studies cited have been conducted at different times and under different market conditions, ${ }^{6}$ and partly as a result, not all base-case scenarios project similar levels of future gas prices. On the other hand, it deserves note that high gas price scenarios do not have a uniform meaning across the studies reviewed here, i.e., they are not systematically defined in a rigorous way to represent a certain point on the plausible price distribution. Therefore, one should not place undue emphasis on the differential between the high- and base-case scenarios.

\subsection{Reference Case Comparisons}

We begin by comparing the Working Group’s Reference Case Scenario to other non-EIA reference case scenarios, drawing on the EIA's own forecast comparisons contained in each Annual Energy Outlook. Results from AEO 2004 shown in Figure 1 suggest that the AEO 2004 Reference case is higher than most other non-EIA reference cases, and therefore that the proposed High Fuel Price Scenario would appear even more aggressive against the alternative

\footnotetext{
${ }^{4}$ For example, see Table 3 of Energy Information Administration. 2002. "Derivatives and Risk Management in the Petroleum, Natural Gas, and Electricity Industries.” SR/SMG/2002-01. October 2002, Washington, DC.

${ }^{5}$ To facilitate comparison, and seek consistency with EIA conventions, published price forecasts have been converted (where necessary and possible) to 2002 \$/Mcf terms using: (a) the GDP Chain-Type Price Index (1996=1.0) contained in AEO 2004; (b) a conversion factor of 1.019 MMBtu/Mcf; and (c) the EIA's estimate that Henry Hub prices have been, on average, 10.8\% higher than wellhead prices (where wellhead prices represent the national average price received by natural gas producers for marketed gas, as reported on Form EIA-895).

${ }^{6}$ For example, the gas prices projected in each scenario are highly dependent upon supply assumptions. As such, differences in price forecasts could merely reflect different supply assumptions, rather than inherent differences in response or the models employed. This paper does not compare the supply assumptions embedded in the different scenarios.
} 
reference cases shown. Because Figure 1 is focused solely on reference cases, however, it provides limited information on alternatives to "base-case" scenarios. ${ }^{7}$

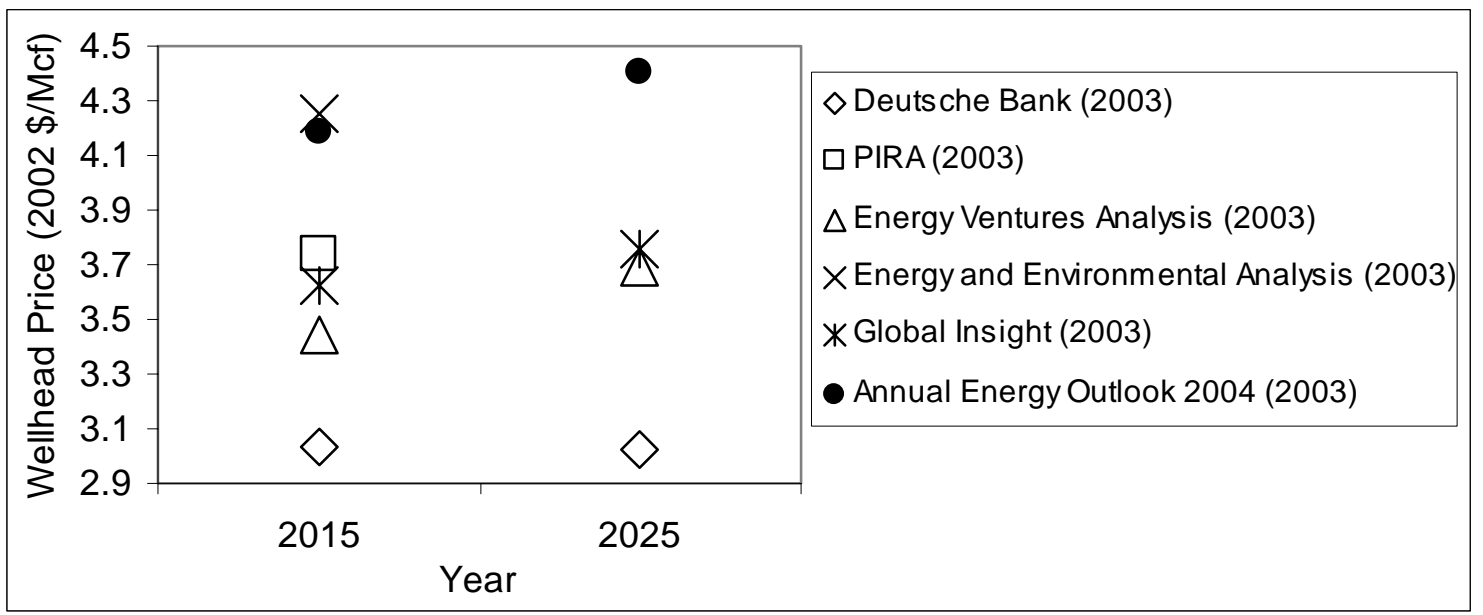

Figure 1. AEO 2004 Natural Gas Price Forecast Comparisons (2015 and 2020)

\subsection{EIA High Gas Price Scenarios}

In addition to generating its own Reference case (which, as noted earlier, is also the Working Group's proposed Reference Case Scenario), the EIA routinely develops high natural gas price scenarios. For example, in AEO 2004 there are three scenarios that typically produce higher natural gas prices than the EIA's Reference case - the High Oil Price case, the High Economic Growth case, and the Slow Oil \& Gas Technological Progress case. Figure 2 shows that wellhead gas prices in the EIA’s Slow Oil \& Gas Technological Progress case are \$0.69/Mcf above the EIA's Reference case in 2025.

In February 2004 the EIA released a report titled Analysis of Restricted Natural Gas Supply Cases, in which it examined, both individually and combined, scenarios involving no Alaskan gas pipeline, low LNG imports, and low unconventional gas recovery relative to the AEO 2004 Reference case. The Combined Low Supply case is shown in Figure 2. Wellhead prices are projected to be $\$ 1.21 / \mathrm{Mcf}$ higher than the EIA Reference case in 2025, and consistent in absolute magnitude (at $\sim \$ 5.5 / \mathrm{Mcf}$ ) with the price level proposed for 2025 by the Working Group. ${ }^{8}$

\footnotetext{
${ }^{7}$ Other reference cases in addition to those shown also exist. For example, the International Energy Agency’s (IEA) World Energy Outlook 2000 projects US wellhead gas prices to be \$3.2/Mcf in 2010 and \$4.5/Mcf in 2020 (both in 2002 dollars).

${ }^{8}$ Additional older EIA publications have also generated high gas price forecasts, but given the existence of more recent projections (see Figure 2) and the run-up in gas prices in recent years, we have decided not to include these older, outdated forecasts. It should also be noted that the EIA's high and low gas price cases are not attempts to bound the limits of uncertainty. Instead, they merely represent plausible alternative scenarios designed to provide some insight into the reaction of energy markets to higher fuel prices.
} 




Figure 2. Natural Gas Prices Resulting from Different EIA Scenarios (based on AEO 2004)

\subsection{Other High Gas Price Scenarios}

A number of private-sector forecasting services generate proprietary high gas price scenarios that are available only for a fee; we did not seek to obtain such forecasts. We were, however, able to find and review a number of non-EIA gas price forecasts and scenarios that are in the public domain.

In the fall of 2003, at the request of Secretary of Energy Spencer Abraham, the National Petroleum Council (NPC) released a landmark study examining the domestic natural gas market. The study modeled two broad scenarios, titled the Reactive Path and the Balanced Future scenarios. Figure 3 shows the range of potential gas prices projected in the NPC study by modeling runs from Energy and Environmental Analysis (EEA). In 2025, the upper bound wellhead price (representing extremes of the Reactive Path scenario, at over \$6.5/Mcf) is well above that proposed by the Working Group, and is roughly $\$ 1.9 / \mathrm{Mcf}$ above the mid-point of the full range of projected prices. ${ }^{9}$

\footnotetext{
${ }^{9}$ Though simplistic, it is not entirely unreasonable to consider the mid-point of the range to represent the "most likely” or reference case price estimate.
} 




Figure 3. National Petroleum Council Gas Price Scenarios

Also in the fall of 2003, the National Commission on Energy Policy (NCEP) commissioned EEA to model gas prices under various scenarios. Figure 4 shows the key results, presented in a report titled Increasing U.S. Natural Gas Supplies. In 2025, wellhead gas prices in the High Gas Price scenario are $\sim$ \$6/Mcf, \$1.8/Mcf above NCEP’s Reference scenario.

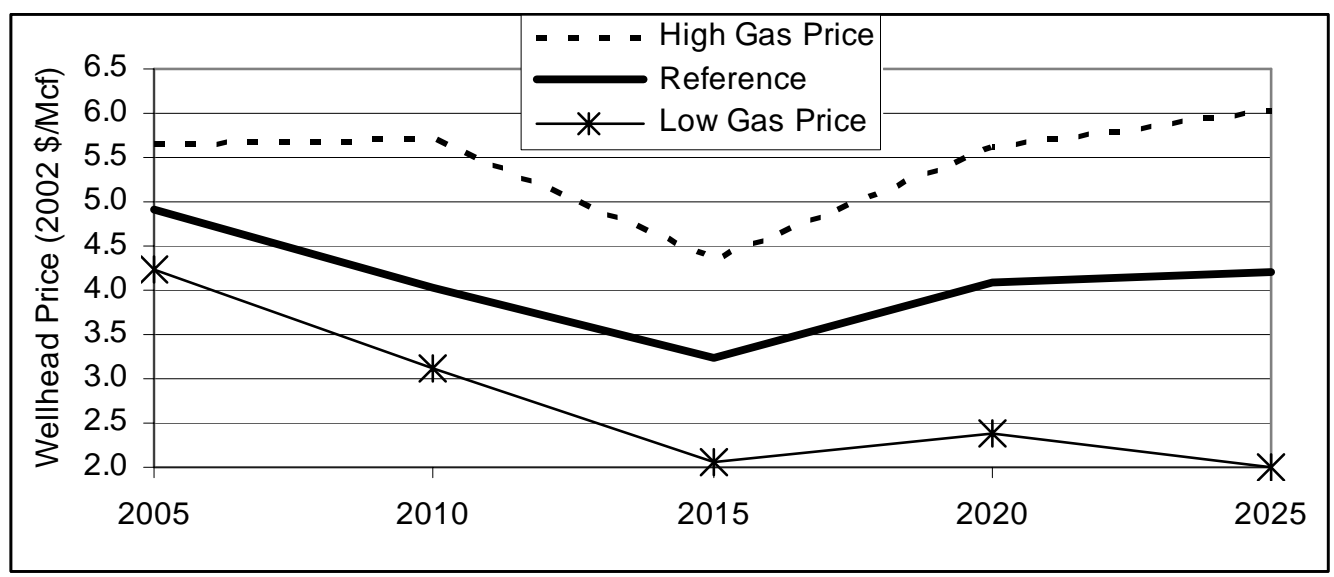

Figure 4. NCEP Gas Price Scenarios

In April 2003, the Northwest Power Planning Council (NWPPC) published revised natural gas price forecasts as inputs to it Fifth Power Plan. As shown in Figure 5, the High gas price scenario is just under \$4.5/Mcf in 2025, \$0.68/Mcf above the Medium scenario in 2025. 




Figure 5. NWPPC Gas Price Scenarios

Stanford's Energy Modeling Forum (EMF) recently concluded (in 2003) a study on natural gas markets in the U.S. (EMF 20). Figure 6 presents wellhead gas prices in 2020 resulting from seven different scenarios run on seven different models. Each scenario to the right of the Low Supply + High Demand scenario builds off of the Low Supply + High Demand scenario. For example, the High Oil scenario uses the Low Supply + High Demand scenario as a base, and layers on high oil prices. ${ }^{10}$ The High Oil scenario - which seems to produce the highest wellhead natural gas prices - is, on average, $\$ 1.1 / \mathrm{Mcf}$ higher than the Reference case in 2020 (EMF 20 results do not extend to 2025).

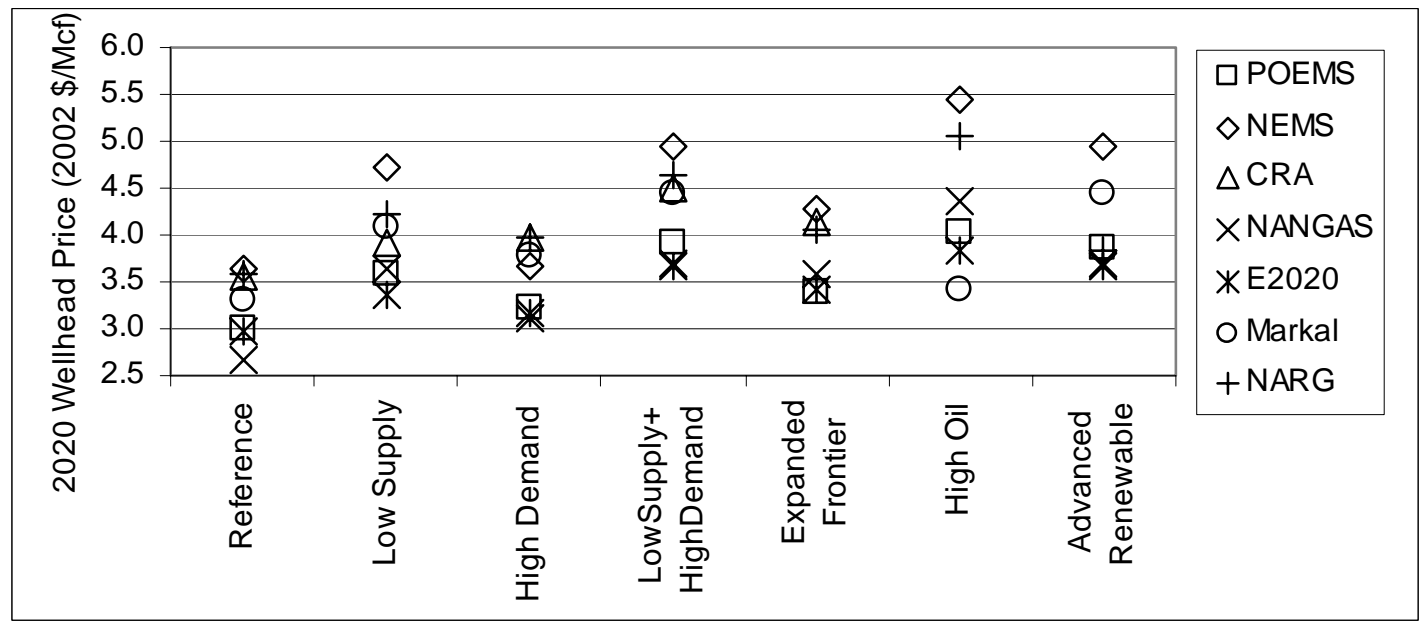

Figure 6. Natural Gas Price Forecasts from Stanford Energy Modeling Forum (EMF 20)

In its 2004 report titled A Balanced Energy Plan for the Interior West, Western Resource Advocates (in conjunction with Synapse Energy Economics) established base case and high gas

\footnotetext{
10 The EMF adopted the EIA's AEO 2003 Reference case oil price forecast as its High Oil prices. The EMF Reference case, meanwhile, uses oil prices from the EIA's AEO 2003 low oil price case.
} 
price forecasts. Both the Business as Usual and Balanced Energy Plan scenarios start with the 2002 delivered natural gas price and then escalate that price at the rates used in AEO 2003. In the base case, gas prices therefore rise from around \$3.65/Mcf (in 2002\$) in 2004 (which is well below the current price of natural gas) to $\$ 5.20 / \mathrm{Mcf}$ by 2020 . To account for fuel price risk, the study examines a high gas price scenario in which natural gas prices are $25 \%$ higher than the base case described above, yielding a 2020 price of $\$ 6.50 /$ Mcf, which is $\$ 1.30 /$ Mcf higher than the base case in that year.

The California Energy Commission (CEC), in a December 2003 report $^{11}$, also estimates lower 48 average wellhead gas prices under a range of scenarios through 2013 (using the North American Regional Natural Gas, or NARG, model). The Base Case forecast rises to $\$ 3.8 /$ Mcf (2002\$) by 2013, while the CEC’s Plausible Low and High cases reach \$3.2/Mcf and \$5.2/Mcf, respectively. Differences between the High gas price and Base Case forecast reach \$1.4/Mcf by 2013.

Hanson et al. ${ }^{12}$ project a variety of energy futures for the United States using scenario analysis and the AMIGA model developed by Argonne National Laboratory. Four alternative, integrated scenarios are developed: the official future, cheap energy reigns supreme, big problems ahead, and technology drives the market. The official future and big problems ahead scenarios effectively represent reference case and high fossil price scenarios, respectively, but in both cases these are integrated scenarios developed by altering numerous input assumptions, not just fuel price inputs. In addition, the big problems ahead scenario is intended to represent a chaotic, event-driven scenario as opposed to a "smooth" scenario typical of most energy models. Unlike the other scenarios presented in this paper, the Hanson et al. scenarios extend until 2050. The average wellhead natural gas prices in the two scenarios are presented below. Differences in gas prices between the two scenarios range from \$0.57/Mcf to \$1.32/Mcf.

Table 1. Wellhead Gas Prices in Two Hanson et al. Scenarios

\begin{tabular}{|l|c|c|c|}
\hline Scenario & $\mathbf{2 0 1 0}$ & $\mathbf{2 0 2 0}$ & $\mathbf{2 0 5 0}$ \\
\hline Official Future (2002\$/Mcf) & $\$ 2.81$ & $\$ 2.97$ & $\$ 5.50$ \\
\hline Big Problems Ahead (2002\$/Mcf) & $\$ 4.13$ & $\$ 3.54$ & $\$ 6.39$ \\
\hline
\end{tabular}

\subsection{Scenarios from Utility Integrated Resource Plans}

We also examined several recent utility integrated resource plans (IRPs). Idaho Power's 2004 IRP includes three gas price scenarios. As shown in Figure 7, the wellhead price in the High gas price scenario is \$1.43/Mcf above the Expected gas price scenario in 2025 (at \$5.2/Mcf), and Idaho Power gave this high price scenario a $30 \%$ probability of occurrence.

\footnotetext{
${ }^{11}$ California Energy Commission. 2003. “Electricity and Natural Gas Assessment Report.” 100-03-014F. Sacramento, Calif.: California Energy Commission.

${ }^{12}$ Hanson, D., I. Mintzer, J. Laitner and J. Leonard. 2004. "Engines of Growth: Energy Challenges, Opportunities, and Uncertainties In the $21^{\text {st }}$ Century.” Argonne, Ill.: Argonne National Laboratory.
} 


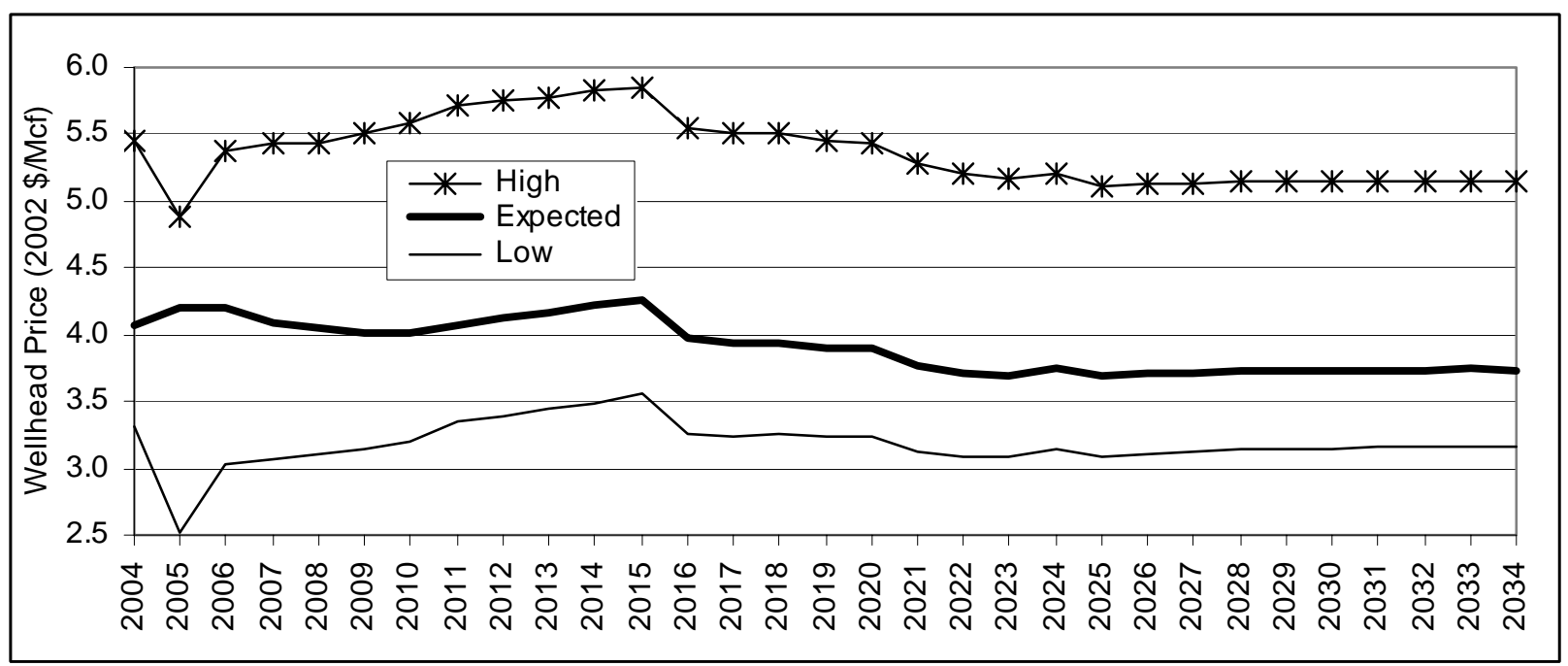

Figure 7. Natural Gas Price Forecasts from Idaho Power's 2004 Integrated Resource Plan

Avista Utilities uses Monte Carlo simulation rather than scenarios to account for uncertainty in future gas prices. To reflect the lognormal distribution of gas prices (i.e., capped on the downside at \$0, while potentially unlimited on the upside), Avista's 2003 IRP assumes that natural gas prices have an asymmetrical distribution. Gas prices are also inversely correlated with hydroelectric generation - as hydropower falls, gas prices increase. Figure 8, taken from Avista's 2003 IRP, shows the resulting gas prices from 200 Monte Carlo simulations (the prices in Figure 8 are presumably nominal, and for delivery of gas to a Northwest trading hub, though this is not clearly stated in the IRP). Note that towards the end of the forecast period the $80 \%$ confidence interval - whose bounds could reasonably be considered a high and low gas price scenario - is roughly $\$ 3 / \mathrm{MCf}$ wide (i.e., $\sim \$ 1.5 / \mathrm{Mcf}$ above and below the average price). In addition, Avista analyzed several additional scenarios, including a high gas price scenario of twice the average price listed in Figure 8 (when translated into 2002\$/Mcf, this stress case is $\sim \$ 3.5 /$ Mcf greater than the average price in 2023). 


\section{Annual Wholesale Natural Gas Prices \\ 2004-2023}

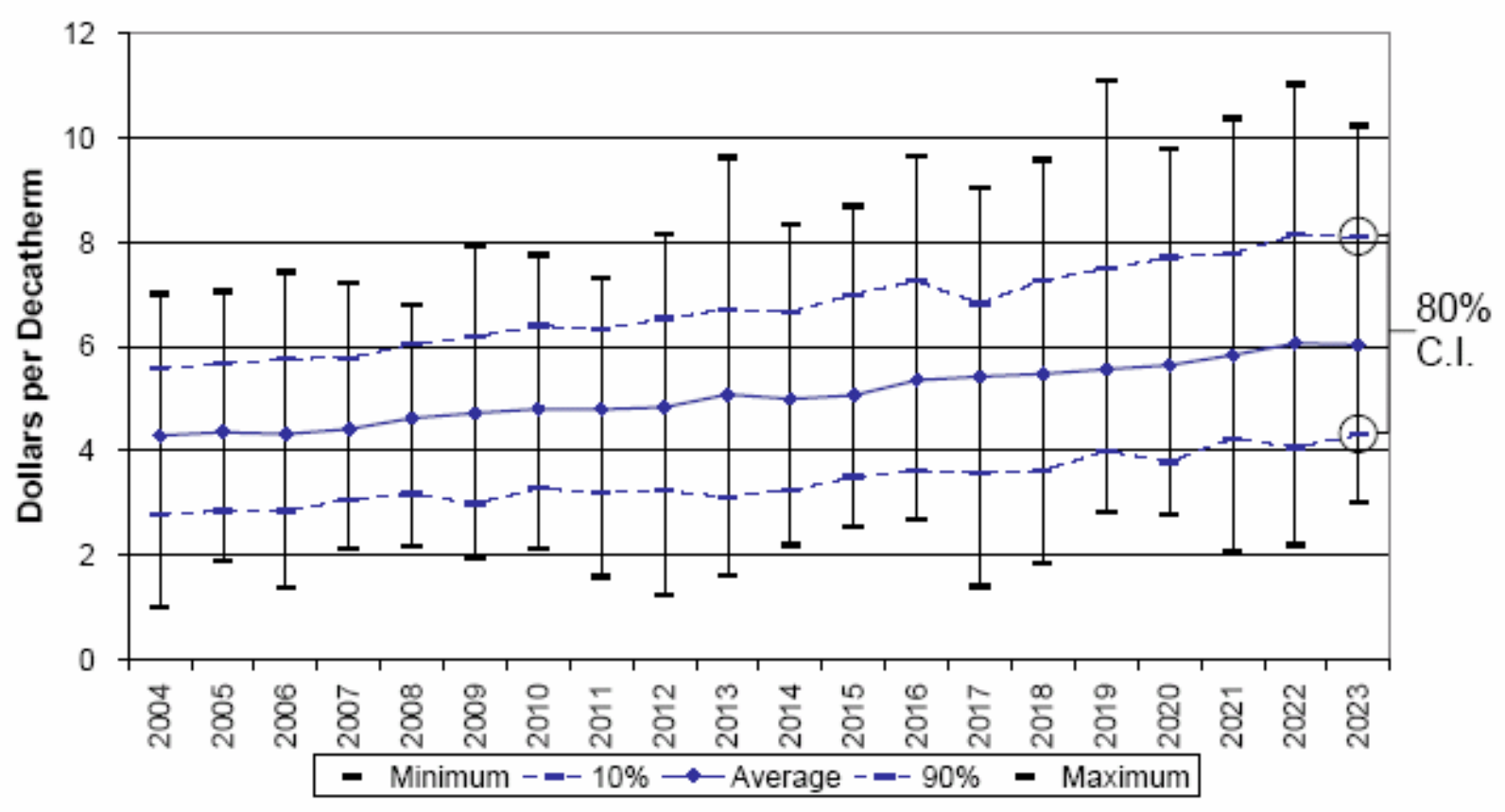

Figure 8. Avista Utilities Reference Case Gas Price Forecast (from Avista’s 2003 IRP)

Northwestern Energy's 2004 IRP relies upon the NWPPC's Medium gas price forecast (see Figure 5 above), and conducts scenarios in a range from $80 \%$ to $130 \%$ of that forecast. On the high end (130\%), this yields a 2025 price of $\$ 4.88 / \mathrm{Mcf}$, which is $\$ 1.13 / \mathrm{Mcf}$ above the Medium base case.

Nevada Power's 2004 IRP contains Low, Base, and High natural gas price forecasts through 2023, with the High case exceeding the Base case by over \$1/Mcf. Nevada Power also analyzed the impact of a one-year gas price shock of over $\$ 4 /$ Mcf relative to the Base case scenario.

The Xcel/PSCo 2004 IRP applied four gas commodity price scenarios of \$3, \$4, \$5, and \$6/Mcf, yielding a $\$ 1.5 / \mathrm{Mcf}$ difference between the medium and high forecast values.

IRP's from other utilities that we reviewed provided little additional information of relevance. ${ }^{13}$

\footnotetext{
${ }^{13}$ For example, in an update to its 2003 IRP, Puget Sound Energy (PSE) averages four gas price forecasts - two from Cambridge Energy Research Associates (CERA), one from Petroleum Industry Research Associates (PIRA), and the NWPPC's Medium scenario shown above. The IRP provides graphs, but not tables, of these gas price projections in nominal dollars for Northwestern trading hubs. Given the difficulty in deciphering and translating the data behind these graphs into a form that would enable comparison within this report, we instead simply note here that PSE adopted as its “extreme” (high) gas price scenario NWPPC's Medium gas price forecast, which is shown in Figure 5 above. The average natural gas price forecast contained in Portland General Electric's 2004 IRP Final Action Plan was also constructed using natural gas bid prices and the NWPPC forecast. Monte Carlo analysis was conducted around these values. PGE's high price forecast simply matched the AEO 2004 reference case assumptions. PacifiCorp performed Monte Carlo analysis of projected natural gas prices, with parameters derived
} 


\subsection{Past EIA Forecast Accuracy}

Finally, we look at the past accuracy of EIA Reference case natural gas price projections, going back to Annual Energy Outlook 1985. Figure 9 illustrates that EIA Reference case gas price projections have historically been trend-following, lagging behind movements in spot market prices (note that this graph is in nominal \$/Mcf, whereas others presented in this section have been normalized to 2002 \$/Mcf where possible). As such, the EIA over-forecast gas prices throughout much of the 1980's and 1990's (when actual gas prices fell and remained relatively low and steady), and more recently appears to have under-forecast gas prices (as actual gas prices have risen). The dark hatched line represents the current (i.e., from August 11, 2004) 6year forward price curve from the NYMEX futures market (adjusted to wellhead-equivalent and \$/Mcf per footnote 5). Note that this forward curve is well above Reference case forecasts from recent AEO's, and is arguably a better projection of spot gas prices over the short- to intermediate-term than the EIA Reference case forecast (assuming that the forward curve reflects the market's expectation of future prices). ${ }^{14}$

Overall, this picture of past forecast accuracy and variability should give pause to those who believe that gas price forecasts have narrow uncertainty bounds. First, expectations of future gas prices have clearly varied substantially over time. From 1997 to 2004, for example, EIA Reference case expectations for year 2015 gas prices rose from just over \$3/MCF to over \$5/MCF. From 1986 to 1997, however, expected prices for the year 2000 dropped from over \$7/Mcf to approximately \$2/Mcf. With such wide variations in expected future gas prices over time, very wide uncertainty bounds on future gas prices are warranted. Second, as is clear from Figure 9, the historic accuracy of long-term gas price forecasts is poor. Forecasts from the 1980's clearly overestimated future gas prices by wide margins, while forecasts from the late 1990's to the present appear to have underestimated prices. If we apply the error seen in past forecasts to current forecasts, sizable uncertainty bounds of at least plus-or-minus $\$ 2 / \mathrm{Mcf}$ appear to have merit.

from historic data and econometric analysis. Average prices were established based on PacifiCorp's internal forward curve and PIRA's March 2002 long-term forecast.

${ }^{14}$ It bears mention here that the EIA's Reference case forecasts are intended to serve as baselines for policy analysis, and are not intended to capture the impact of a variety of events that can cause price volatility in markets. Indeed, all EIA Reference case forecasts assume no change in current policy over the forecast period - an assumption that is bound to render such forecasts inaccurate. Nonetheless, Figure 9 (and Figure 14 later) paints a clear picture of the tremendous uncertainty that exists in forecasts of natural gas (and oil) prices. 


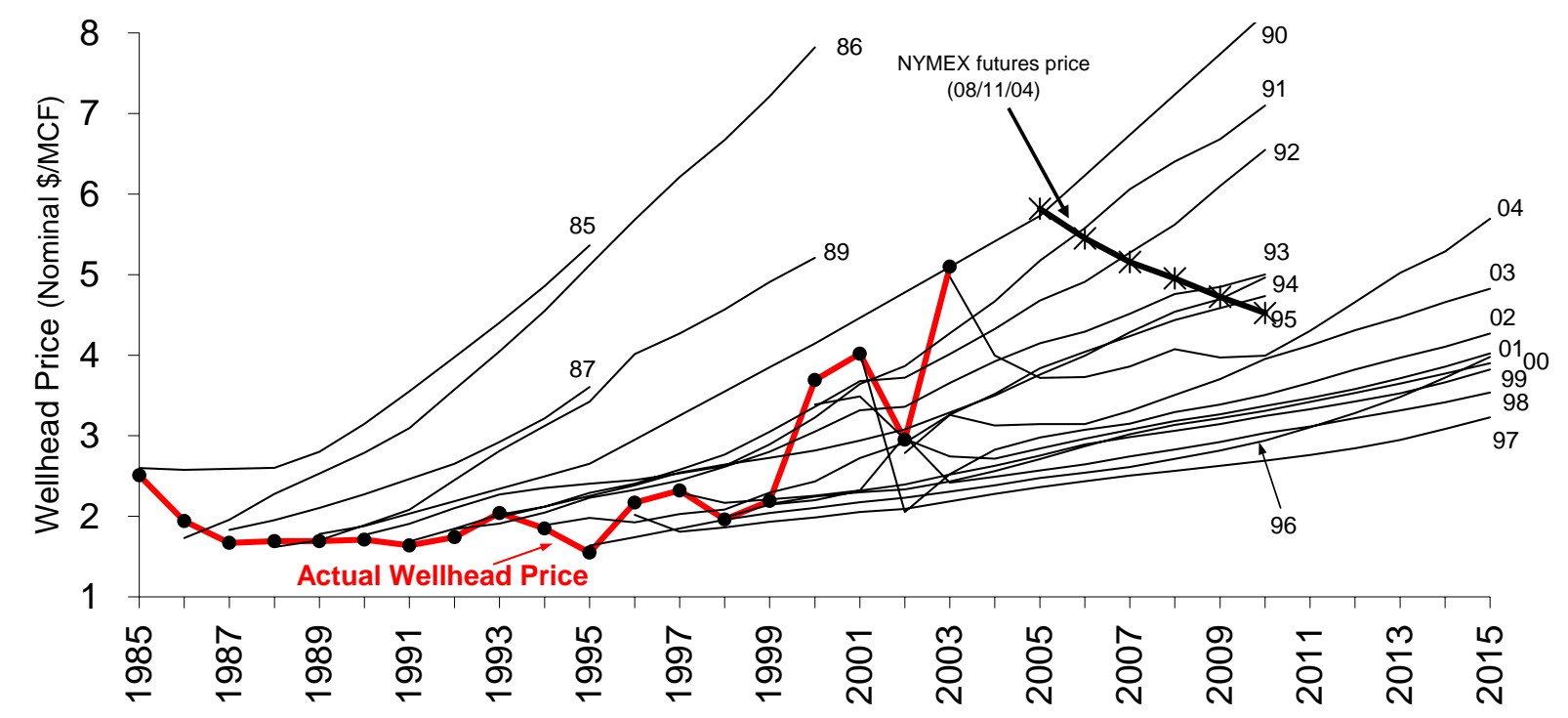

Source: EIA

Figure 9. Past EIA Natural Gas Price Reference Case Forecast Accuracy

\subsection{Summary}

In conclusion, the natural gas prices through 2025 proposed in the FE-EERE Scenarios Working Group's High Fuel Price Scenario appear to be reasonable based on current natural gas prices and other externally generated gas price forecasts and scenarios. If anything, an even more extreme gas price scenario could be considered.

Specifically, the difference in year 2025 between the proposed High Fuel Price Scenario and Reference Case Scenario equals \$1.07/Mcf. This differential is well within the range exhibited by other scenarios presented above, i.e., the high gas price scenarios range from $\$ 0.50-\$ 2.00 / \mathrm{Mcf}$ above the corresponding reference case prices in 2020 or 2025. In addition, at least four of the scenarios shown above, the NPC Reactive Path and NCEP High Gas Price scenarios, the EIA's Combined Low Supply case, and Western Resource Advocates' high gas price scenario, exhibit gas prices in excess of \$5.50/Mcf in 2025 (the price achieved in that year of the proposed High Fuel Price Scenario). Finally, as noted earlier, the past accuracy of EIA's Reference case forecasts suggests reasonable uncertainty bounds of at least \$2/Mcf.

The price escalation from $\$ 5.50$ in 2025 to $\$ 7.50$ by 2050 within the proposed High Fuel Price Scenario is harder to evaluate, primarily because few existing forecasts or scenarios extend beyond 2025. As shown earlier in Table 1, however, Hanson et al. do project prices to 2050, and show prices in that year that are $\$ 2.50-\$ 2.75 / \mathrm{Mcf}$ higher than in 2020 . This compares favorably with a difference of \$2.00/Mcf (\$7.50/Mcf in 2050 less \$5.50/Mcf in 2025) in the proposed High Fuel Price scenario. Moreover, given the long-term nature of this extended forecast (i.e., to 2050), increasing demand for natural gas among all sectors of the economy, and the finite amount of recoverable natural gas in the world, a price as aggressive as $\$ 7.50 / \mathrm{Mcf}$ by 2050 does not seem beyond reason, particularly for a High Fuel Price Scenario. 


\section{Oil Price Scenarios}

Like natural gas prices, future oil prices are also highly uncertain, and have proven difficult to predict, especially given that both economic influences and OPEC behavior are in play. In its memorandum dated July 30, 2004, the FE-EERE Scenarios Working Group proposed a High Fuel Price Scenario whereby world oil prices would follow the path described in the EIA's High Oil Price case. While the Working Group expressed a desire to gain access to and use the High Oil Price case that will be included in AEO 2005, that scenario is not yet available, and instead we use the High Oil Price case from AEO 2004 in this document for comparison purposes. We note, however, that EERE and FE staff have asked the Working Group to develop a more aggressive high oil price scenario than that envisioned in the AEO (and given the trend in oil prices this year, a higher oil price is expected in the AEO 2005 than appeared in AEO 2004); as such, our comparisons to the AEO 2004 High Oil Price case should be considered indicative, at best.

The Working Group's proposed high oil price scenario, at least as initially linked to the AEO 2004 High Oil Price case, contains world oil prices (in $2002 \$ / b b l$ ) that reach $\$ 33.00$ by 2010, $\$ 34.00$ by 2015, $\$ 35.00$ by 2025, and $\$ 45.00$ by 2050 . In comparison, the Working Group’s proposed Reference Case Scenario (which is simply EIA's Reference case as of April 9, 2004) shows world oil prices reaching $\$ 24.17$ by 2010, $\$ 26.02$ by 2020, and $\$ 27.00$ by 2025 (the ultimate year of the EIA's Reference case forecast). Thus, in 2025, world oil prices in the initially proposed High Fuel Price Scenario (based on AEO 2004) are \$8.00/bbl above oil prices in the proposed Reference Case Scenario.

In this section, we benchmark the reasonableness of the oil prices contained in the initially proposed High Fuel Price Scenario against other published scenarios. Where possible, we report not only the absolute price level of each scenario, but also the degree to which any high oil price scenario exceeds the corresponding "base-case" scenario, comparing that differential to the gap between the Working Group’s proposed High Fuel Price and Reference Case scenarios.

\subsection{Reference Case Comparisons}

We begin by comparing the AEO 2004 Reference case to other non-EIA reference cases, drawing on the EIA's own forecast comparisons contained in each Annual Energy Outlook. Results from AEO 2004 shown in Figure 10 suggest that the AEO 2004 Reference case is higher than most other non-EIA reference cases, and therefore that the Working Group's proposed High Fuel Price Scenario would appear even more aggressive against the alternative reference cases shown. ${ }^{15}$ Because Figure 10 is focused solely on reference cases, however, it does little to inform us of alternatives to "base-case" scenarios.

\footnotetext{
${ }^{15}$ Note that definitional differences may account for some of the variation in base-case forecasts shown in Figure 10. For example, as noted in AEO 2004, "The world oil price measure varies by forecast. In some it is the spot price for West Texas Intermediate (WTI), Brent, or a basket of crude oils. AEO2004 uses the composite U.S. refiners' acquisition cost of crude oil, including transportation and fees. There is no simple way to put the forecasts for oil prices...on a common basis.”
} 


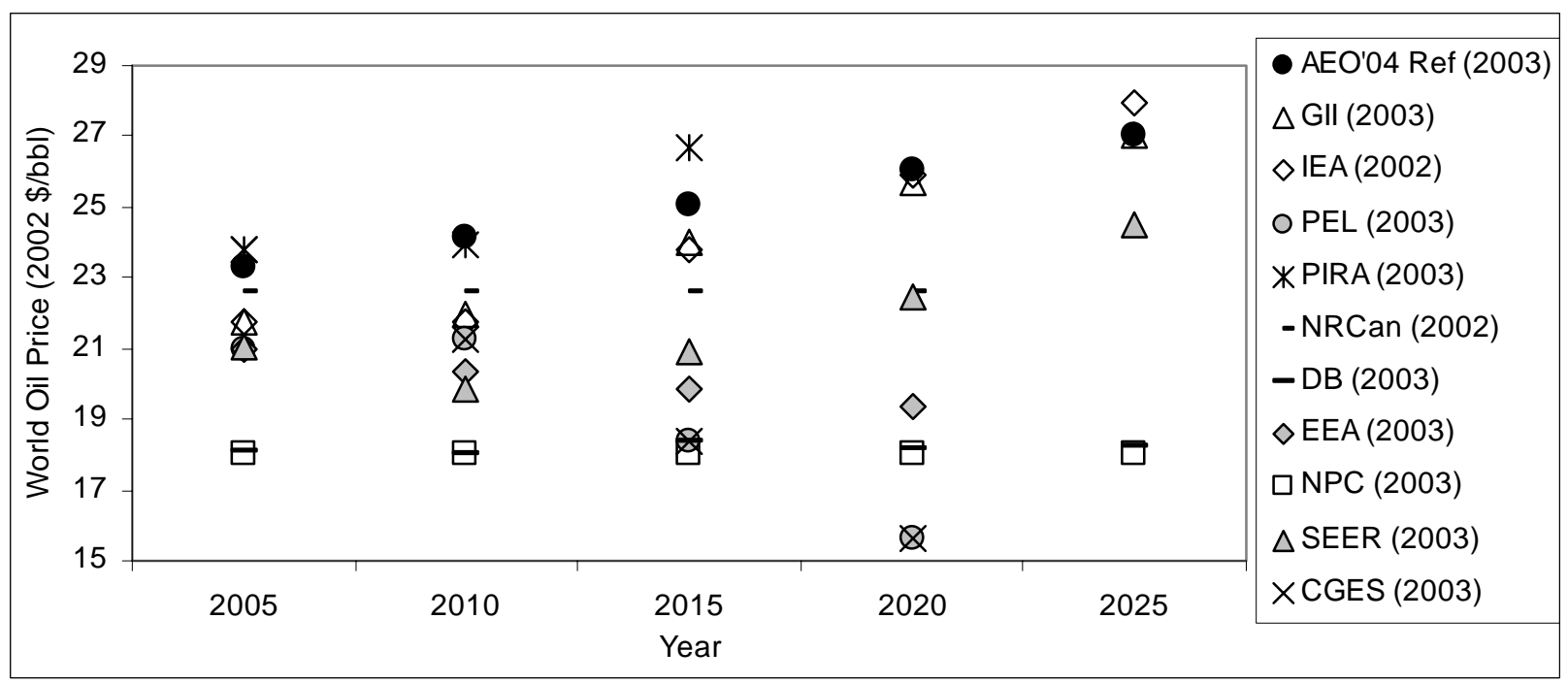

Figure 10. AEO 2004 Oil Price Forecast Comparisons

\subsection{EIA High Oil Price Scenarios}

The EIA has also developed several high oil price scenarios. For example, in AEO 2004 there are two scenarios that produce higher oil prices than the Reference case - the High Oil Price case and the High Economic Growth case (recall that the AEO High Oil Price case was initially proposed by the Working Group as part of its High Fuel Price Scenario). In addition, AEO 2004 contains a Low Oil Price case. Figure 11 confirms that the High Oil Price case in particular is $\$ 8.00 / \mathrm{bbl}$ above the Reference case in $2025 .^{16}$

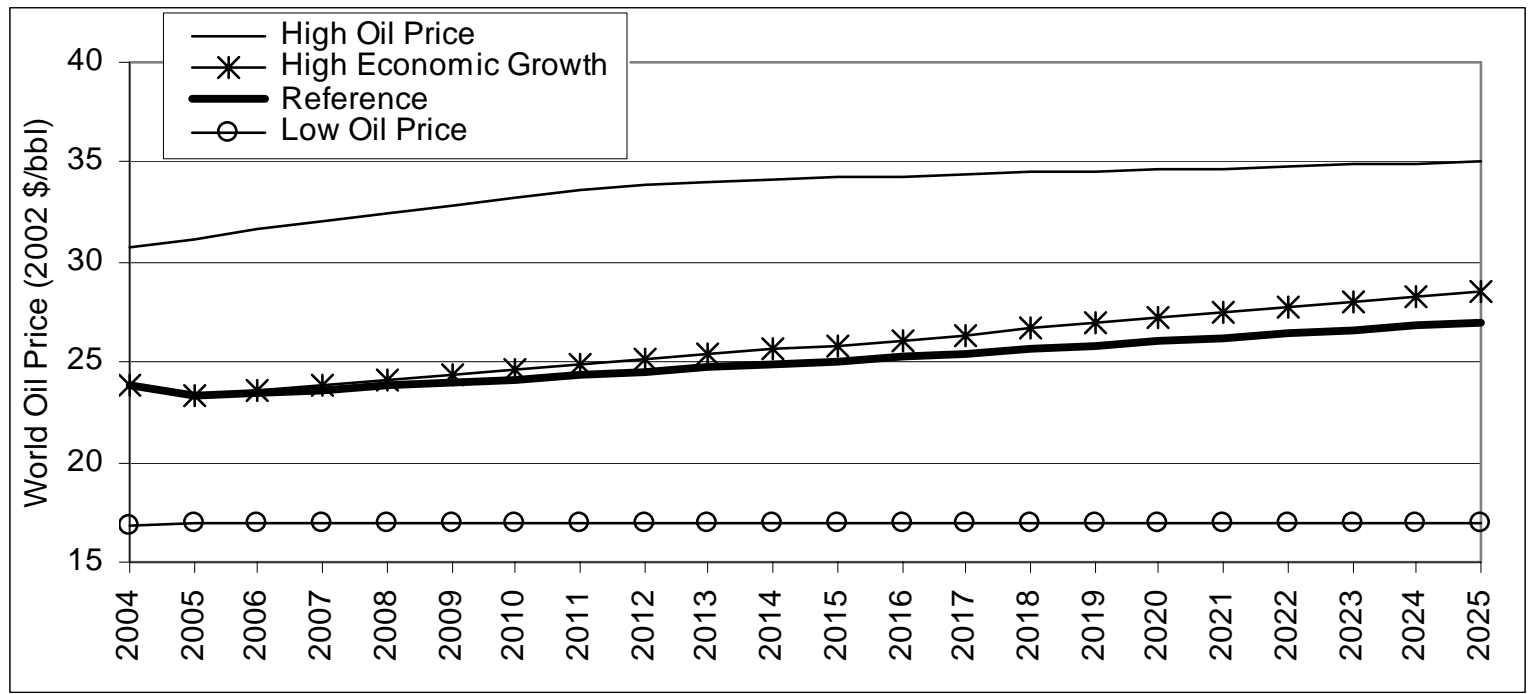

Figure 11. AEO 2004 Oil Price Scenarios

\footnotetext{
${ }^{16}$ It should be noted that the EIA's High and Low Oil Price cases are not attempts to bound the limits of uncertainty. Instead, they merely represent plausible alternative scenarios designed to provide some insight into the reaction of energy markets to higher fuel prices.
} 


\subsection{Other High Oil Price Scenarios}

A number of private-sector forecasting services generate proprietary high oil price scenarios that are available only for a fee; we did not seek to obtain such forecasts. We were, however, able to find and review a number of non-EIA oil price forecasts and scenarios that are in the public domain.

In April 2003, the Northwest Power Planning Council (NWPPC) generated oil price forecasts as inputs to it Fifth Power Plan. As shown in Figure 12, the High oil price scenario is $\$ 8.18 / \mathrm{bbl}$ above the Medium scenario in 2025. This differential, as well as the absolute level of prices contained in the High scenario, are quite similar to those shown above from AEO 2004.

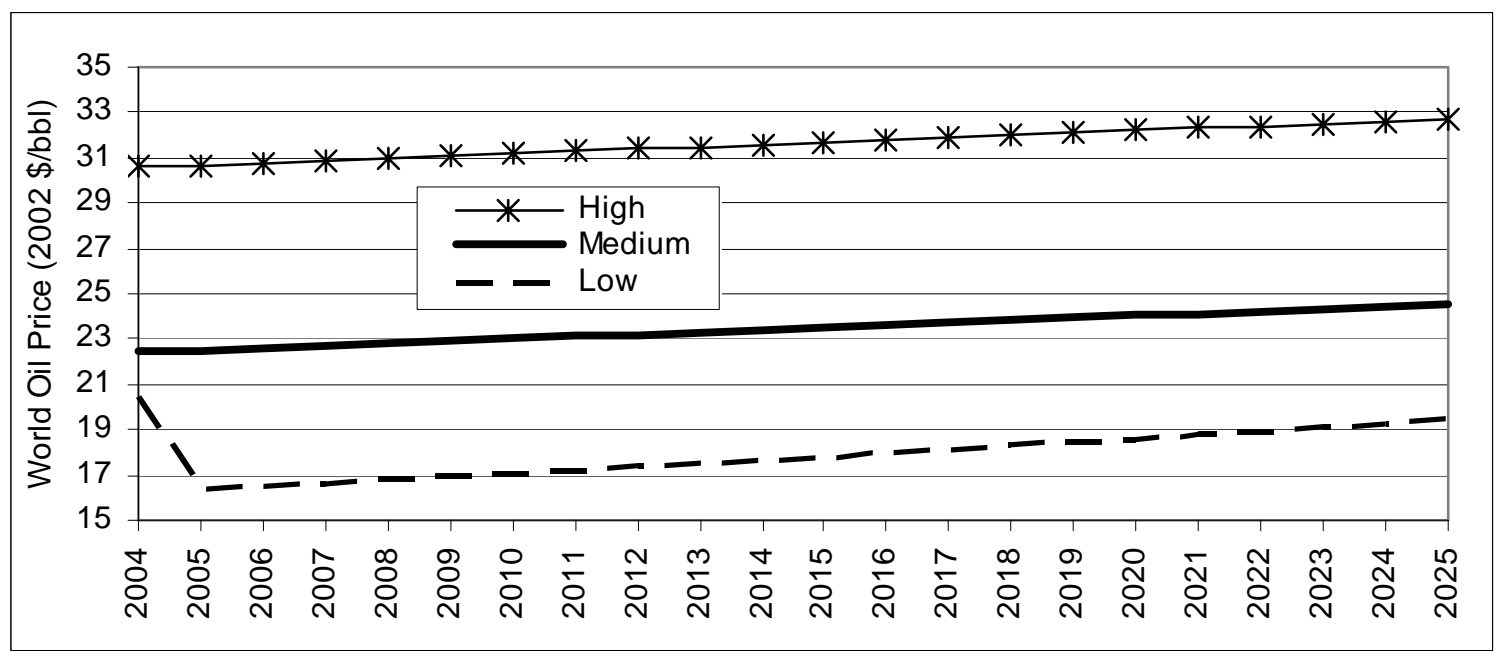

Figure 12. NWPPC Oil Prices for Fifth Power Plan

In 2003, Stanford's Energy Modeling Forum concluded a study on natural gas markets in the U.S. (EMF 20), which included a single High Oil Price scenario. The EMF adopted the EIA's AEO 2003 Reference case oil price forecast as its High Oil prices. The EMF Reference case, meanwhile, simply uses oil prices from the EIA's AEO 2003 Low Oil Price case. Figure 13 presents world oil prices in 2020 assumed in this scenario, along with the reference scenario, from five different models. The EMF 20 High Oil scenario is, on average, $\$ 7.36 / \mathrm{bbl}$ higher than the reference case in 2020 (EMF 20 results do not extend to 2025). 




Figure 13. EMF 20 High Oil Price and Reference Scenarios

As described in the previous section, Hanson et al. (2004) project a variety of energy futures for the United States using scenario analysis and the AMIGA model developed by Argonne National Laboratory. Two of these scenarios, the official future and big problems ahead scenarios, effectively represent reference case and high fossil price scenarios. The big problems ahead scenario is specifically intended to represent a chaotic, event-driven world. The average world oil price in the two scenarios is presented below in Table 2. Differences in world oil prices between the two scenarios range widely, from \$36/bbl in 2010 and \$23/bbl in 2020, to \$14/bbl by 2050 .

Table 2. World Oil Prices in Two Hanson et al. Scenarios

\begin{tabular}{|l|c|c|c|}
\hline Scenario & $\mathbf{2 0 1 0}$ & $\mathbf{2 0 2 0}$ & $\mathbf{2 0 5 0}$ \\
\hline Official Future $(2002 \$ / \mathrm{bbl})$ & $\$ 23.92$ & $\$ 24.84$ & $\$ 27.30$ \\
\hline Big Problems Ahead $(2002 \$ / \mathrm{bbl})$ & $\$ 59.91$ & $\$ 47.64$ & $\$ 41.41$ \\
\hline
\end{tabular}

A 1997 Delphi survey of experts in the oil industry conducted by the California Energy Commission generated low, most likely, and high oil price forecasts out to $2018 .{ }^{17}$ In 2018 , prices were expected to be $\$ 18 / \mathrm{bbl}, \$ 26 / \mathrm{bbl}$, and $\$ 43 / \mathrm{bbl}$ respectively (all in 2002 dollars). Thus, the differential between the high and most likely scenarios was about $\$ 17 / \mathrm{bbl}$ in 2018. Although this survey is dated, we include its results here as an alternative to forecasts generated by constrained energy models. It is specifically worth noting that the experts in the Delphi survey apparently view future oil prices as more uncertain than seen in most energy models, i.e., the difference between the high and most likely scenarios in the Delphi survey are greater than shown by the energy models presented earlier.

\subsection{Past EIA Forecast Accuracy}

Finally, we look at the past accuracy of EIA reference case oil price projections, going back to Annual Energy Outlook 1985. Figure 14 illustrates that EIA reference case oil price projections have historically been trend-following, lagging behind movements in spot market prices (note

\footnotetext{
${ }^{17}$ California Energy Commission. 1998. "Results of Delphi IX Survey of Oil Price Forecasts.” Sacramento, Calif.: California Energy Commission. http://www.energy.ca.gov/reports/DELPHI-9.PDF
} 
that this graph is in nominal $\$ / \mathrm{bbl}$, whereas others presented in this section have been normalized to 2002 \$/bbl where possible). As such, the EIA over-forecast oil prices throughout much of the 1980's and 1990's (when actual oil prices fell and remained relatively low and steady), and more recently appears to have under-forecast oil prices (as actual oil prices have risen, and currently hover around $\$ 45-50 / \mathrm{bbl}$ ). The dark hatched line represents the current (i.e., from August 11, 2004) 6-year forward price curve from the NYMEX futures market. Note that this forward curve is well above reference case forecasts from recent AEO's, and is arguably a better projection of spot oil prices over the short- to intermediate-term than the AEO's Reference case forecast (assuming that the forward curve reflects the market's expectation of future prices). ${ }^{18}$

As with the natural gas results presented earlier, this picture of past forecast accuracy and variability should give pause to those who believe that oil price forecasts have narrow uncertainty bounds.

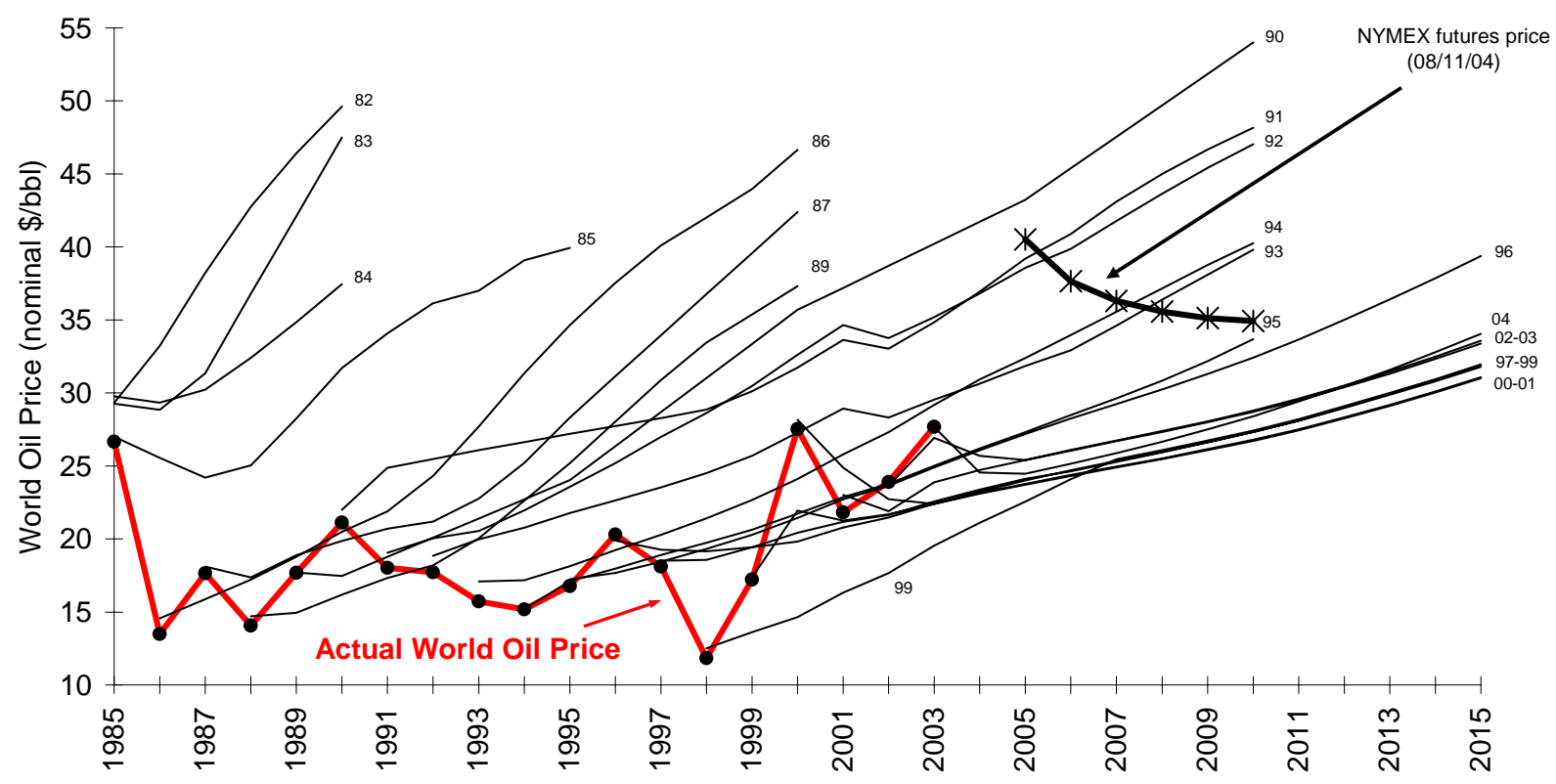

Figure 14. Past EIA Oil Price Reference Case Forecast Accuracy

\subsection{Summary}

In conclusion, the oil prices originally proposed by the FE-EERE Working Group in the High Fuel Price Scenario (assuming AEO 04) appear to be reasonable, if not too low, based on: (1) the forward market for oil, which predicts prices to average in the $\$ 35-40 / \mathrm{bbl}$ range for 6 years, (2) current prices which, while representing a peak and not an annual average, at \$45-50/bbl have already reached the projected price based on the AEO 2004 High Oil Price case in 2050,

\footnotetext{
${ }^{18}$ Again, we acknowledge that EIA Reference case forecasts are not intended to capture the impact of a variety of events that can cause price volatility in markets. We also note that NYMEX oil futures prices, which are deliverable to Cushing, Oklahoma, are not directly comparable to the world oil prices used by the EIA, which reflect average refiner acquisition costs for imported crude oil. Thus, the comparison between the NYMEX futures prices and world oil prices in Figure 14 should be considered to be illustrative only.
} 
(3) other externally generated oil price forecasts and scenarios, and (4) the historical difficulty in accurately forecasting future oil prices.

Specifically, the difference in year 2025 between the Working Group's originally proposed High Fuel Price Scenario and Reference Case Scenario equals \$8.00/bbl (based on AEO 2004). This margin is at the low end of the range of differences exhibited by other scenarios which, in the order presented above, equal \$8.18/bbl (in 2025), \$7.36/bbl (in 2020), \$23/bbl (also in 2020), and $\$ 17 / \mathrm{bbl}$ (in 2018).

In fact, with oil prices currently hovering around $\$ 45-50 / \mathrm{bbl}$ and many geologists predicting the end of "cheap" oil as they expect production from economically recoverable reserves to peak in the near future, one could potentially argue that adopting the prices contained in the EIA's AEO 04 High Oil Price case may not be extreme enough for the purpose at hand. It is expected, however, that the AEO 2005 High Oil Price case will be more aggressive, and may not be subject to the same degree of criticism. Overall, a spread between the oil prices in the FE-EERE High Fuel Price and Reference Case Scenarios of well over \$8/bbl is supported by the literature. In fact, based on recent FE and EERE staff guidance, the Working Group has been exploring a more aggressive oil price scenario than originally envisioned. 


\section{Carbon Regulation Scenarios}

The development of domestic carbon regulation in the 2005-2050 timeframe is possible, if not likely, though the actual design, timing, and magnitude of those regulations are uncertain.

Several bills that would regulate carbon emissions are currently pending before the U.S. Congress, most notably the Climate Stewardship Act (S. 139, McCain-Lieberman) ${ }^{19}{ }^{19}$ While the U.S. does not plan to ratify the Kyoto Protocol, ratification by a sufficient number of other countries may result in Kyoto coming into force.$^{20}$ Finally, even if one believes that national carbon regulation in the U.S. is unlikely, state-level action has already begun and many expect state-level action to intensify. As such, in the 2005-2050 timeframe, some level of carbon regulation is a near certainty (at least in a subset of U.S. states).

The FE-EERE Scenarios Working Group has proposed a Carbon Cap-\&-Trade Scenario that involves a simple carbon cap for all energy sectors. Under the scenario, U.S. carbon emissions are to be reduced from the baseline by approximately 500 million metric tons of elemental carbon equivalent (mmtce) by 2017. Carbon reductions are assumed to begin in 2010. After 2017, the cap is held constant through 2050, which is equivalent to stabilizing U.S. carbon emissions at about 1996 levels (1470 mmtce). Because of the complexity involved in modeling such a scenario, the Working Group further proposed that carbon banking not be considered, that nuclear capacity be held constant, that no international offsets be employed, and that actual regulatory details be ignored. ${ }^{21}$

\subsection{EIA Carbon Policy Scenarios}

EIA has itself analyzed several sets of proposed carbon regulations:

Kyoto: In October 1998, EIA published The Impacts of the Kyoto Protocol on U.S. Energy Markets and Economic Activity. The study analyzed the commitment of the United States under Kyoto to limit carbon emissions to 7\% below 1990 levels in the 2008-2012 timeframe (on an average basis). Because the specific rules under Kyoto had not yet been established, the EIA study evaluated multiple carbon reduction scenarios, as shown in Figure 15. In each case, the analysis assumed that the target would remain constant from 2013 to 2020 (the end of the forecast period), and the targets were assumed to have a three year phase in period, beginning in 2005. EIA's analysis of the cost of these carbon reduction scenarios shows a wide range of results, from $\$ 67$ per metric ton of carbon equivalent (mtce) to $\$ 348$ per metric ton (1996\$) depending on the stringency of the carbon cap. EIA performed sensitivity cases based on variations in economic growth, technology improvement, and nuclear additions. EIA also compared their results to those of other comparable analyses, finding that the EIA's analysis

\footnotetext{
${ }^{19}$ Other carbon-related bills that have been considered recently include H.R. 4067, S. 366, H.R. 2042, S. 843, H.R. 3093.

${ }^{20}$ Currently, only ratification by Russia is necessary to allow Kyoto to come into force.

${ }^{21}$ Of course, ignoring carbon banking and offsets will likely increase the cost of compliance with the carbon capand-trade program. This suggests that the Working Group should retain some flexibility to incorporate such mechanisms in the event that the resulting cost of compliance is found to be prohibitive, and hence not a likely future legislative outcome.
} 
projects a higher cost of carbon reduction than the other models analyzed. EIA's 1990-7\% results assume that international trading and sinks are not allowed; all carbon reductions must occur domestically in the energy sector. The other cases evaluated by EIA were intended to reflect a plausible range of costs under alternative scenarios that might involve greater flexibility in achieving the Kyoto targets (including the use of sinks and international trade in carbon credits). The Working Group’s proposed Carbon Cap-\&-Trade Scenario, with an approximate 500 mmtce reduction of energy-sector carbon emissions by 2017, is less stringent than one of the Kyoto scenarios analyzed by EIA, but is more aggressive than the others (shown in Figure 15).

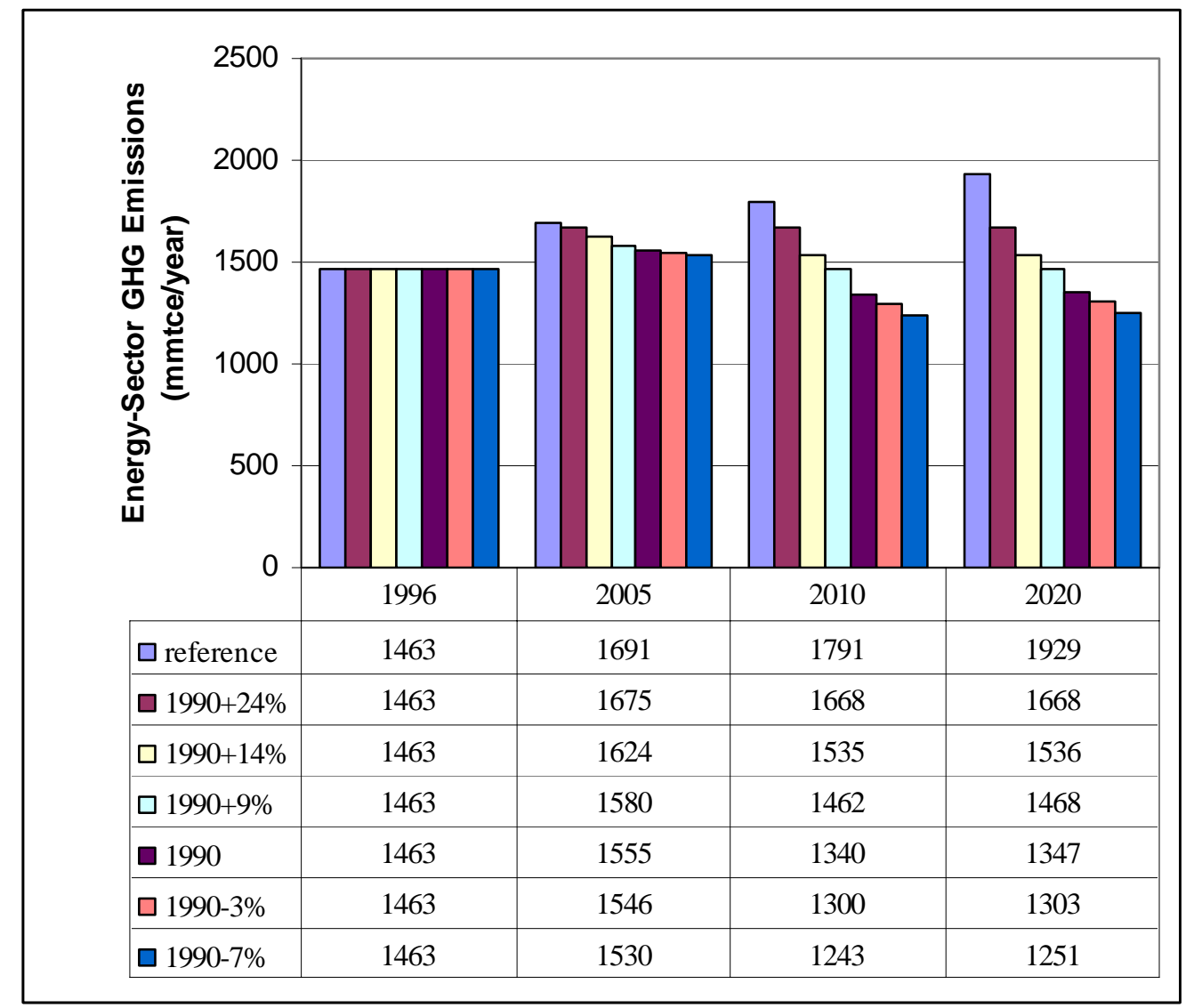

Figure 15. EIA's Analysis of the Kyoto Protocol

Climate Stewardship Act: In May 2004, the EIA released an update to its June 2003 study of the Climate Stewardship Act of 2003 (S.139, McCain-Lieberman). The May 2004 updated analysis reflects the impact of a proposed amendment to the original bill (offered by the bill's authors, Senators McCain and Lieberman, in SA.2028) that, among other things, removes reference to a more restricted second phase emission cap starting in 2016, but leaves the original 2010 cap intact (namely, limiting GHG to 2000 levels by 2010). The EIA's updated analysis projects that under the amended bill (SA.2028), total GHG emissions would be reduced by 152 mmtce in 2010, $413 \mathrm{mmtce}$ in 2020, and $624 \mathrm{mmtce}$ in 2025 relative to the reference case (partial results of EIA's reference, S.139, and SA.2028 results are presented in Figure 16). These amounts are broadly comparable to the carbon reductions contained in the Working Group's proposed Carbon Cap-and-Trade Scenario, though it deserves note that the EIA analysis of McCain-Lieberman does include a modest amount of non-energy-sector emissions reductions. 
Allowance costs are $\$ 55 /$ mtce (2001\$) in 2010, $\$ 125 /$ mtce in 2020, and $\$ 167 /$ mtce in $2025 .{ }^{22}$ Unlike the FE-EERE Working Group’s proposed scenario, however, the McCain-Lieberman bill allows banking of carbon allowances as well as the use of a variety of offset allowances. As such, implementation of the Working Group's proposed scenario may result in higher compliance costs and market disruption than the EIA's analysis of the Climate Stewardship Act. On the other hand, the Climate Stewardship Act exempts certain sectors from the carbon regulation, whereas the Working Group has proposed no such restrictions. Finally, it deserves note that EIA has conducted substantial sensitivity analysis on their S.139 results, including a high technology case, a no new nuclear/no sequestration case, a high natural gas price case, a no banking case, a commercial coverage case, varied auction cases, and two different offset cases.

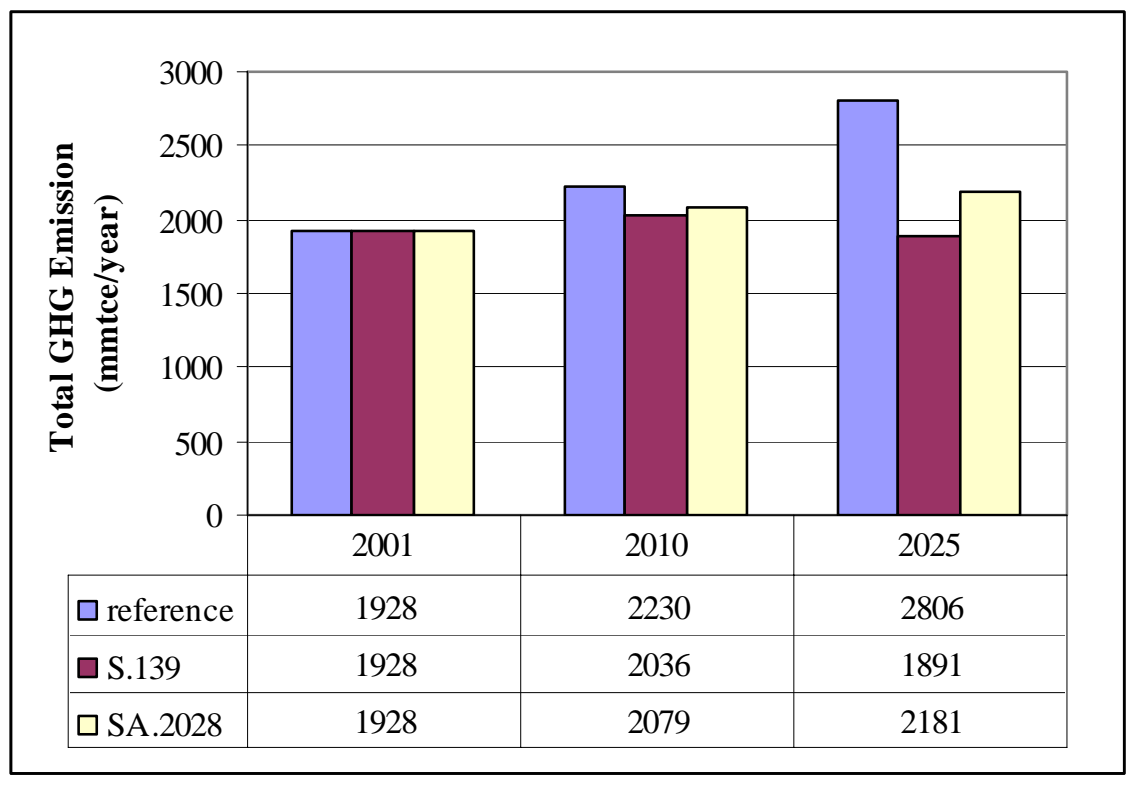

Figure 16. EIA's Analysis of the Climate Stewardship Act

Multi-Pollutant Emissions Reductions: In its 2001 report titled Power Plant Emission Reductions Using a Generation Performance Standard, the EIA compares two different types of cap-and-trade programs to reduce emissions of $\mathrm{CO}_{2}$ and other pollutants. The first approach involves a program patterned after the $\mathrm{SO}_{2}$ allowance program resulting from the 1990 Clean Air Act Amendments (detailed results from these cases are presented in the July 2001 report Analysis of Strategies for Reducing Multiple Emissions from Electric Power Plants: Sulfur Dioxide, Nitrogen Oxides, Carbon Dioxide, and Mercury and a Renewable Portfolio Standard.). The second involves a dynamic generation performance standard (GPS), which is primarily a different means of allocating emissions allowances. Under both approaches, the power sector must reduce $\mathrm{CO}_{2}$ emissions to 1990 levels by 2008 (by 2005 in an alternative case), and then to 7\% below 1990 levels over the period from 2008-2012. These reductions, in percentage terms relative to the reference case, are more aggressive than those proposed by the Working Group. However, this study looked only at power sector carbon emissions, while the Working Group's proposal would apply to the entire energy sector; as such - in terms of aggregate reductions - the

\footnotetext{
${ }^{22}$ Emissions offset prices - at \$89/mtce and \$106/mtce in 2020 and 2025, respectively - are lower than allowance prices, as a $15 \%$ cap on the use of offsets for compliance becomes binding post-2010, resulting in competition to supply the least costly offsets.
} 
Working Group scenario is more aggressive. Results show carbon allowance prices (in 1999\$) ranging from roughly \$130-\$150/mtce in both 2010 and 2020. In a later analysis, titled Analysis of Strategies for Reducing Multiple Emissions from Electric Power Plants with Advanced Technology Scenarios, EIA used more aggressive technology and policy assumptions, resulting in lower projected costs of compliance.

\subsection{Other Domestic Carbon Reduction Scenarios}

More generally, one can benchmark the proposed FE-EERE Scenarios Working Group proposed Carbon Cap-\&-Trade Scenario to other domestic scenarios for carbon reduction, and the cost of achieving that reduction.

- Scenarios of U.S. Carbon Reductions: Potential Impacts of Energy-Efficient and LowCarbon Technologies by 2010 and Beyond. ${ }^{23}$ The 5-Lab study evaluated the potential impacts and costs of aggressive carbon reduction commitments in the U.S. Three scenarios were considered: (1) an efficiency case, (2) a \$25/mtce high-efficiency/low-carbon case, and (3) a \$50/mtce high-efficiency/low-carbon case. The study projects carbon reductions of 120 mmtce by 2010 under the efficiency case, 230 mmtce under the $\$ 25 /$ mtce permit price, and 390 mmtce under the $\$ 50 /$ mtce permit price. In the final instance, carbon emissions were projected to fall to year 1990 levels due to a range of policy instruments, including the imposition of a \$50/mtce tax. In a later analysis, Scenarios for a Clean Energy Future, a similar basket of policies and measures was found to bring carbon emissions to 1990 levels, but only by 2020 and only in the Advanced - \$50/mtce case (the Moderate case and Advanced - \$25/mtce cases do not achieve such deep reductions in carbon emissions).

- The Energy Modeling Forum. The Energy Modeling Forum produced a systematic comparison of 13 modeling analyses of greenhouse gas (GHG) emission reduction costs, using a common set of emissions reduction scenarios and common assumptions for selected parameters. ${ }^{24}$ The EMF results estimate that a tax of $\sim \$ 5-37.5 /$ ton-CO2 ( $\left.\sim 18-\$ 138 / \mathrm{mtce}\right)$ might be required to hold emission at 1990 levels by 2010 (continuing through 2050), assuming no emissions trading. To achieve emission reduction of 7\% below 1990 levels by 2010 (continuing through 2050) required a carbon tax of $\sim \$ 12.5-69 /$ ton-CO2 ( $\$ 46-$ $\$ 253 /$ mtce). International carbon trade is projected to significantly reduce these compliance costs, and a variety of other emissions reduction scenarios (including some longer-term stabilization scenarios) were analyzed.

- McCain-Lieberman. To illustrate the sensitivity of modeling results to input assumptions, it is useful to briefly note the results of multiple analyses of the McCain-Lieberman Climate Stewardship Act, which have been conducted by EIA, MIT, NRDC, and Charles River Associates. These studies derive disparate results - not reviewed here - with carbon permit prices easily varying by a factor of two depending on the study and its underlying assumptions.

\footnotetext{
${ }^{23}$ Interlaboratory Working Group. 1997. "Scenarios of U.S. Carbon Reductions: Potential Impacts of EnergyEfficient and Low-Carbon Technologies by 2010 and Beyond.” Oak Ridge, TN and Berkeley, CA: Oak Ridge National Laboratory and Lawrence Berkeley National Laboratory. ORNL-444 and LBNL-40533. September.

${ }^{24}$ See Weyant, J. 1999. “The Costs of the Kyoto Protocol: A Multi-Model Evaluation.” Special Issue of The Energy Journal.
} 
- President Bush's GHG intensity proposal. In February 2002, President Bush unveiled a proposal to reduce the greenhouse gas (GHG) intensity of the US economy by $18 \%$ over ten years (i.e., by 2012). By the White House's own analysis (see http://www.whitehouse.gov/news/releases/2002/02/climatechange.html), the President's proposal would "achieve 100 million metric tons of reduced emissions in 2012 alone, with more than 500 million metric tons in cumulative savings over the entire decade.” This reportedly represents a $4.5 \%$ reduction in forecasted emissions over this time period, and as such is less ambitious than the cuts called for in the Kyoto Protocol.

- Hanson et al. As described earlier, Hanson et al. (2004) derive four different scenarios of U.S. energy markets over the coming 50 years. The Official Future Scenario results in growth of U.S. carbon emissions from 1559 mmtce in 2000 to 2471 mmtce by 2050. The base case of the Technology Drives the Market Scenario, on the other hand, results in carbon emissions of 1741 mmtce by 2050, while the policy case of the Technology Drives the Market Scenario results in deep reductions of carbon emissions to 839 mmtce.

- Other Analyses: A number of additional advocacy-based analyses of carbon reduction scenarios have also been conducted. In The Path to Carbon Dioxide-Free Power, for example, the Tellus Institute projects that utility-sector carbon emissions by 2020 of half that of year 2004 emissions would be possible through a mix of aggressive renewable energy and energy efficiency policies. ${ }^{25}$ In its Clean Energy Blueprint, meanwhile, the Union of Concerned Scientists also projects that deep reductions in carbon emissions would be possible with aggressive utility-sector energy efficiency and renewable energy policies. In particular, power sector carbon emissions decline to year 1990 levels by 2009, while by 2020 carbon emissions are 47\% below year 1990 levels. ${ }^{26}$ A variety of other, similar analyses, have been prepared by Tellus Institute and other organizations - for example, Energy Innovations (1997) and Policies and Measures to Reduce CO2 in the U.S. (1998) offer policies and measures expected to reduce carbon emissions by $414 \mathrm{mmtce}$ and $593 \mathrm{mmtce}$, respectively, in 2010.

\subsection{International Comparisons}

One can also benchmark the FE-EERE Scenarios Working Group proposed Carbon Cap-\&Trade Scenario with other scenarios of international carbon emissions. For example, in its 2000 Special Report on Emissions Scenarios, the IPCC - using six integrated assessment models common to the literature - developed a set of scenarios to represent the range of driving forces and emissions in the scenario literature. This multi-model approach yielded 40 scenarios that together encompass the current range of uncertainties of future GHG emissions. None of these scenarios explicitly include additional climate initiatives, such as the Kyoto Protocol. Figure 17 presents the range of $\mathrm{CO}_{2}$ emissions scenarios included in the report, normalized to an index where 1990 emissions levels equal one. Although the scenarios presented in Figure 17 are global and not U.S.-specific, they nevertheless serve to highlight (a) the wide range of possible emissions scenarios, (b) that stabilization of carbon emissions post-2017 at 1996 levels (i.e., the

\footnotetext{
${ }^{25}$ Bailie, A., S. Bernow, B. Castelli, P. O’Connor, J. Romm. 2003. "The Path to Carbon Dioxide-Free Power: Switching to Clean Energy in the Utility Sector.” A Study for the World Wildlife Fund.

${ }^{26}$ Clemmer, S., D. Donovan, A. Nogee and J. Deyette. 2001. “Clean Energy Blueprint: A Smarter National Energy Future for Today and the Future.” Union of Concerned Scientists, with the American Council for an EnergyEfficient Economy and the Tellus Institute.
} 
Working Group's proposed Carbon Cap-and-Trade Scenario for the US) is a scenario that falls within the range presented here (again, on a global scale), (c) but that stabilization at 1996 levels by 2017 does represent an aggressive carbon reduction case. That said, it is not clear how much comparison can or should be made between international and U.S. emissions growth, as the dynamics differ considerably for developed and developing countries.

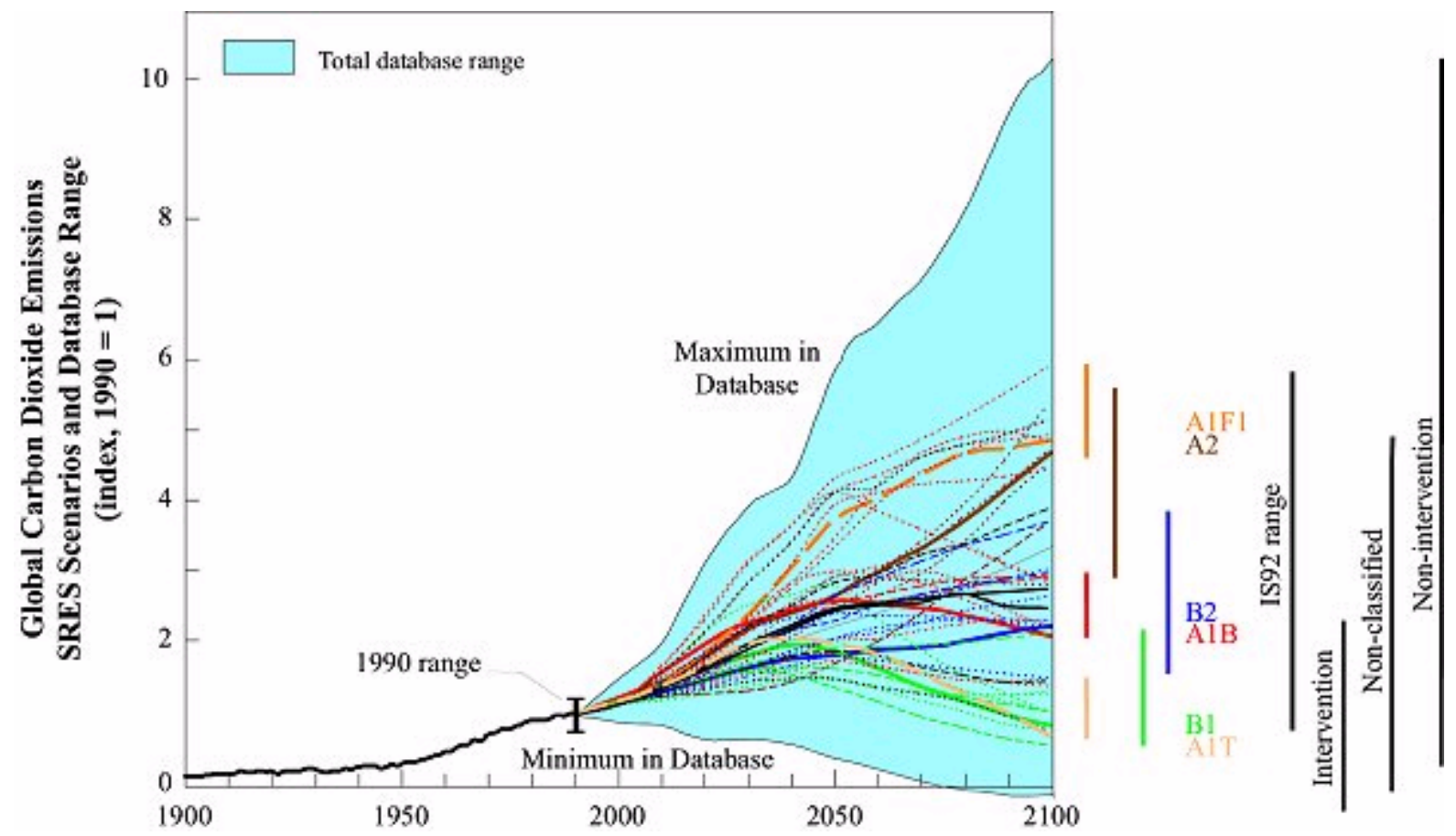

Figure 17. Range of Global $\mathrm{CO}_{2}$ Emissions, IPCC's Special Report on Emissions Scenarios

More generally, a great deal of analysis has been conducted to evaluate the potential impacts of carbon reduction generally, and Kyoto compliance in particular. Substantial disagreement remains on the cost of achieving carbon reductions. Technical-economic (bottom-up) models often project that emissions reductions of $10-30 \%$ may be achievable at little or no net cost to society, while macroeconomic (top-down) models typically conclude that costs will be significant. This is not always the case, however: some macroeconomic general equilibrium models exhibit a high degree of flexibility, which can result in compliance at low costs. Moreover, models such as NEMS, which represent a combination of top-down and bottom-up approaches, can yield compliance costs that vary considerably based on assumptions about consumer behavior.

In a recent summary of some of these studies in Energy Economics, ${ }^{27}$ Springer found that estimated compliance costs varied widely both across models (due to different modeling assumptions and approaches) and under varying assumptions for Kyoto compliance rules (e.g., allowance for international trading, sinks, etc.). Assuming liberal global trading for carbon dioxide credits, permit prices under Kyoto range from $\$ 1$ to $\$ 22$ per metric ton of CO2 (2000\$) depending on the study, with an average of $\$ 9$ (\$3.7-\$81/mtce, with an average of $\$ 33 / \mathrm{mtce})$. If

\footnotetext{
${ }^{27}$ Springer, U. 2003. "The Market for Tradable GHG Permits Under the Kyoto Protocol: A Survey of Model Studies.” Energy Economics 25: 527-551.
} 
trade is allowed only among Annex B countries, however, permit prices under the same Kyoto requirements are projected to range from \$3-74 per metric ton of CO2 (2000\$) depending on the study, with an average of \$27 (\$11-\$271/mtce, with an average of \$99/mtce).

\subsection{Carbon Scenarios in Utility Integrated Resource Planning}

The private sector is also beginning to recognize the risk of future carbon regulation. For example, an increasing number of utility integrated resource plans are describing carbon risks, and analyzing those risks. We review treatment of carbon risk in some of these plans, which are in the public domain, below. Other private energy sector firms, such as oil companies, are also beginning to consider carbon risk, though their assumptions and analysis are typically not in the public domain.

- The PacifiCorp 2003 IRP, for example, modeled a variety of scenarios of possible future cap and trade regulations. PacifiCorp's base-case assumption presumes a carbon cap at year 2000 levels that takes effect in 2009, with allowance costs starting at \$8 per ton of CO2 (\$29/mtce), escalating at 2.5\% per year. Other modeled scenarios include: (1) no cap, (2) $\$ 2 /$ ton-CO2 (\$7.3/mtce) beginning in 2013, with a cap at 2000 levels, (3) $\$ 25 /$ ton-CO2 (\$92/mtce) beginning in 2008, with a cap at 1990 levels, and (4) \$40/ton-CO2 (\$147/mtce) beginning in 2008, with a cap at 1990 levels.

- Idaho Power’s 2004 IRP filing, meanwhile, analyzed carbon regulations equivalent to $\$ 12.30$ per ton of CO2 (\$45/mtce) in the base-case scenario beginning in 2008, and conducted sensitivity analysis on carbon values of $\$ 49.21$ per ton of CO2 ( $\$ 180 / \mathrm{mtce})$ beginning in 2008. Idaho Power estimates a $30 \%$ probability of a zero carbon tax, a $50 \%$ probability of a $\$ 12.30 /$ ton tax, and a $20 \%$ probability of a $\$ 49.21$ tax.

- In Colorado, Xcel/PSCo's 2004 IRP estimated the impact of carbon dioxide regulations at both \$6/ton of CO2 and \$12/ton levels (\$22-\$44/mtce), starting in 2009.

- Portland General Electric’s 2002 IRP analyzed a carbon tax scenario, using a value of \$10 per ton of carbon dioxide (\$37/mtce).

- Avista's 2003 IRP prepared one carbon tax scenario in their IRP, consistent with Northwest Power Planning Council assumptions, with prices of \$1.32 per ton of CO2 in 2004 and increasing to \$11 per ton of CO2 in 2023 (\$5-\$40/mtce).

- In a preliminary draft document released in 2004, the NWPPC has proposed - in its base portfolio analysis - the following probabilities for carbon regulation: zero percent until 2008, 10 percent in 2008, increasing every four years to 50 percent in 2024. Through 2015, offset costs for any future having some level of carbon control range with equal probably from zero to $\$ 15 /$ ton-CO2 (\$55/mtce). Following 2015, the upper bound increases to $\$ 30 /$ ton-CO2 (\$110/mtce). Across all cases, the mean value is \$0.75/ton-CO2 in 2008 (\$2.75/mtce), increasing to \$7.5/ton-CO2 in 2024 (\$27.5/mtce).

- A 2004 California Public Utilities Commission (CPUC) consultant report authored by Energy and Environmental Economics, Inc. also assesses the range of possible future scenarios of carbon regulation and associated costs, and concludes that a conservative and reasonable estimate of carbon costs is a trend of \$5 per ton of $\mathrm{CO} 2$ in the near term, $\$ 12.5 /$ ton by 2008 , and $\$ 17.50 /$ ton by 2013 (the time-discounted value is $\sim \$ 8 /$ ton, or 
\$29/mtce), and that these values should be used in assessing the benefits of energy efficiency investments. $^{28}$

- PG\&E’s 2004 long-term plan, meanwhile, used the present value of the cost trend developed in the CPUC consultant report (\$8/ton-CO2, or $\$ 29 /$ mtce) to estimate the risk reduction value of their preferred generation plan.

\subsection{Conclusions}

Based on the summary presented above, we conclude that a wide range of carbon regulation scenarios are possible, especially within the timeframe considered by EERE and FE (through 2050). The FE-EERE Scenarios Working Group’s Carbon Cap-\&-Trade Scenario is found to be less aggressive than many Kyoto-style targets, somewhat similar in magnitude to the Climate Stewardship Act, and less aggressive that some other scenarios found in the literature.

We also find that the 2010 start-date of the proposed carbon regulation, with full compliance reached in 2017, is conservative relative to other scenarios that have been considered in the literature. ${ }^{29}$ It deserves note that most of the studies presented here emphasize the possibility of near- to medium-term response to climate change, but even more aggressive longer-term carbon reductions might be considered. Such reductions (beyond stabilization at year 1996 levels) are not contemplated in the Working Group proposal, and arguably, should be.

Despite this, the Working Group proposal for carbon reductions is aggressive in the near term, and may explore the limits of NEMS in analyzing the cost of carbon reductions (NEMS, like most energy models, is arguably better at predicting the impact of small deviations in conditions than the impact of sizable market changes). Specifically, we are somewhat concerned that the stringency of the proposed carbon regulation scenario, which ignores carbon banking and offsets and does not allow nuclear power to expand, will lead to a particularly high estimated cost of carbon reduction. If this is true, some will certainly argue that NEMS is not an adequate tool to estimate the cost of stringent carbon regulations. Just as important, in the face of such possible costs, it might be argued that carbon regulations of the type contemplated are politically unlikely. We therefore encourage some flexibility in the Working Group's ultimate implementation of the Carbon Cap-and-Trade Scenario.

\footnotetext{
${ }^{28}$ Energy and Environmental Economics and Rocky Mountain Institute. 2004. "A Forecast of Cost Effectiveness, Avoided Costs and Externality Adders.” Prepared for the California Public Utilities Commission.

${ }^{29}$ On the other hand, given that the earliest that any such regulation or legislation could possibly be enacted would be in 2005, and that prudence would dictate allowing ample time (e.g., possibly several years) to prepare for implementation, 2010 does not seem overly conservative outside of the context of other studies and scenarios examined.
} 


\section{Other Observations}

Here we conclude with three additional (yet somewhat tangential) observations:

1. Additional Scenarios to Consider: While we believe that carbon and high fuel price scenarios are entirely appropriate, our review of other scenarios suggests that at least three additional scenarios merit consideration in future rounds of this work: (1) multi-pollutant regulations that involve increasingly stringent requirements for criteria air pollutants, ${ }^{30}$ (2) external state or national renewable energy and energy efficiency standards (e.g., national RPS, more aggressive state-level RPS programs, more stringent CAFÉ standards), ${ }^{31}$ and (3) inclusion of high coal prices within the High Fuel Price Scenario. The second of these three scenarios, in particular, appears both possible and also highly relevant to EERE's R\&D portfolio, though its interaction with possibly heightened national-level appliance efficiency standards requires greater thought (the application of such standards might be considered an appropriate scenario, but appliance standards are also under the direct responsibility of DOE).

2. Specification of the High Fuel Price Scenario: We are somewhat concerned about the specification of the Working Group's proposed High Fuel Price Scenario. This scenario envisions a target for natural gas prices rising to $\$ 7.5 / \mathrm{mmBTU}$ by 2050 , while oil prices were initially proposed to be set according to EIA's AEO High World Oil Price case. It should be noted, however, that oil and gas prices (as well as coal prices, as substitutes for both oil and gas can be derived from coal, and coal directly competes with gas in the power sector) are linked in energy models, such as NEMS and MARKAL, due to substitution possibilities between the two energy sources. As such, it may simply be impossible to specify a joint gas/oil scenario in the way envisioned above. This consideration should be addressed by the modeling and scenario-building teams.

3. Ability of Integrated Energy Models to Respond to the Scenarios: It deserves note that many analysts have questioned the ability of integrated energy models to adequately respond to extreme scenarios, given that model parameters tend to be set based on historical data that fall within fairly narrow ranges. This debate is particularly acute with carbon regulation, where estimates of the cost carbon reduction range widely, but similar concerns exist in specifying high fuel price scenarios that significantly exceed historical prices. As such, implementing extreme carbon or fuel price scenarios in NEMS or MARKAL (or any other energy model) will require substantial forethought and planning, as well as careful ex-post assessment of the degree to which the model has adequately or appropriately responded to the scenario at hand.

\footnotetext{
${ }^{30}$ Previous studies of the impact of multi-pollutant legislation, however, suggest that retrofitting for pollution control is generally a cheaper option (with the notable exception of carbon) than deploying renewables or energy efficiency, so this scenario may not be particularly interesting to EERE.

${ }^{31}$ Since the renewable or efficiency standards would be present in both the program (with R\&D) and baseline (without R\&D) cases, the degree of renewable or efficiency penetration would most likely be similar in both cases, and the benefits of EERE's R\&D program would be reflected through lower compliance costs (rather than different penetration levels).
} 